\title{
A Pareto-based Evolutionary Algorithm using Decomposition and Truncation for Dynamic Multi-objective Optimization
}

\author{
Junwei Ou${ }^{\mathrm{a}, \mathrm{b}}$, Jinhua Zheng ${ }^{\mathrm{a}, \mathrm{c}, *}$, Gan Ruan ${ }^{\mathrm{d}}$, Yaru $\mathrm{Hu}^{\mathrm{a}}$, Juan Zou ${ }^{\mathrm{a}}$, Miqing \\ $\mathrm{Li}^{\mathrm{d}}$, Shengxiang Yang ${ }^{\mathrm{e}}, \mathrm{Xu} \operatorname{Tan}^{\mathrm{b}, *}$ \\ ${ }^{a}$ Key Laboratory of Intelligent Computing and Information Processing, Ministry of \\ Education, Information Engineering College of Xiangtan University, Xiangtan, Hunan \\ Province, China \\ ${ }^{b}$ School of Software Engineering, Shenzhen Institute of Information Technology, Shenzhen \\ 518172, China \\ ${ }^{c}$ Hunan Provincial Key Laboratory of Intelligent Information Processing and Application, \\ Hengyang Normal University, Hengyang, 421002, China \\ ${ }^{d}$ School of Computer Science, University of Birmingham, Birmingham B15 2TT, U. K. \\ e School of Computer Science and Informatics, De Montfort University, Leicester LE1 9BH, \\ U.K.
}

\begin{abstract}
Maintaining a balance between convergence and diversity of the population in the objective space has been widely recognized as the main challenge when solving problems with two or more conflicting objectives. This is added by another difficulty of tracking the Pareto optimal solutions set(POS) and/or the Pareto optimal front(POF) in dynamic scenarios. Confronting these two issues, this paper proposes a Pareto-based evolutionary algorithm using decomposition and truncation to address such dynamic multi-objective optimization problems (DMOPs). The proposed algorithm includes three contributions: a novel mating selection strategy, an efficient environmental selection technique and an effective dynamic response mechanism. The mating selection considers the decomposition-based method to select two promising mating parents with good diversity and convergence. The environmental selection presents a modified
\end{abstract}

\footnotetext{
* Corresponding author

Email addresses: junweiou@163.com (Junwei Ou), jhzheng@xtu.edu.cn (Jinhua Zheng), GXR847@cs.bham.ac.uk (Gan Ruan), huyaru1199@gmaile.com (Yaru Hu),

zoujuan@xtu.edu.cn (Juan Zou), limitsing@gmail.com (Miqing Li), syang@dmu.ac.uk (Shengxiang Yang), tanxu_nudt@yahoo.com (Xu Tan)
} 
truncation method to preserve good diversity. The dynamic response mechanism is evoked to produce some solutions with good diversity and convergence whenever an environmental change is detected. In the experimental studies, a range of dynamic multi-objective benchmark problems with different characteristics were carried out to evaluate the performance of the proposed method. The experimental results demonstrate that the method is very competitive in terms of convergence and diversity, as well as in response speed to the changes, when compared with six other state-of-the-art methods.

Keywords:

Dynamic multi-objective optimization, Evolutionary algorithms, Decomposition, Diversity

\section{Introduction}

Over the past decades, evolutionary multi-objective optimization (EMO) has been of interest to researchers due to the inherent characteristics of evolutionary algorithms (EAs) when addressing problems with no less than two conflicting 5 goals. Specifically, EAs are able to find a set of trade-off solutions that approximate to the POF. Therefore, multi-objective optimization evolutionary algorithms (MOEAs) have been widely applied in many real-world engineering scenarios [1]. The main challenge for MOEAs in dealing with multi-objective optimization problems (MOPs) is determining how to balance diversity and convergence 1 during the optimization process.

A particular kind of real-life MOPs, called dynamic multi-objective optimization problems (DMOPs), have objective functions, constraints and/or parameters that may be time variant [2]. DMOPs pose considerable challenges to optimization algorithms due to the dynamism of various problems [3] [4] [5].

15 Moreover, the change frequency and change severity are two important param-

\footnotetext{
${ }^{1}$ Convergence and diversity in the paper refer to the objective space, except where explicitly stated otherwise.
} 
eters that play an important role in influencing the performance of DMOEAs [3] 6]. The change frequency [2] defines the number of generations from one environmental change to the next. High severity of change 2] requires algorithms that have good ability to search because when the POS changes in the dynamic environment, the population may lose the ability to trace the changing POS. Even though MOEAs have great advantages for solving MOPs, they also have limitations in solving these problems. The reason is that the values of the objective functions may vary when there are environmental changes. Thus, MOEAs are supposed to be greatly improved to quickly find the POS or POF before the next environmental change 7] 8] comes. In recent years, dynamic multi-objective evolutionary algorithms (DMOEAs) have been extensively applied in many areas, such as scheduling [9] [10], control [11] 12] 13], planning [14] 15] 16\|17|18], design [19] and machine learning 20]. Although traditional MOEAs [21] 22] 23] dealing with MOPs can accelerate the converging speed of

30 the population, one drawback in solving DMOPs is that they sometimes lack adequate diversified solutions to help the population jump out of the current optimum.

Additionally, detection of whether a change has occurred is a critical part during the evolutionary process. Reevaluating solutions [9] [3] [8] 24] [7] 6] and 35 checking the population's statistical information [2] [25] [26] are two main ways to detect a change. The approach of reevaluating solutions is employed by reevaluating members at every generation. Although this approach is easy to implement, it needs additional function evaluations. Checking the population's statistical information is a good way as it doesn't need function evaluation40 s. However, it can cause false positives for detector changes. Jiang et al. [4] introduced a steady-state manner to detect changes. In the manner, the population's individuals are checked in random order one by one to determine whether a discrepancy exists between their previous objective values. If a discrepancy is found, the change is successfully detected and the rest of the population's mem-

45 bers do not need to be checked. However, the population members also need to be reevaluated. Hence, this paper, the approach of reevaluating solutions is 
selected to detect the environmental change. Moreover, less detectable environmental changes [27] pose a big challenge for DMOEAs. We can not deal with such issues now and leave it as one of our future works. The main contributions 50 of this study are summarized as follows.

1. An effective mating selection method was developed to select well converged and diversified parents, and the main goal was to generate good offsprings.

2. In environmental selection process, an improved truncation method was to improve the diversity of whole population.

3. In order to quickly react to environmental change, a good change response mechanism based an exploration strategy and an exploitation strategy was designed.

The paper is structured as follows. Section 2 describes some basic definitions, related works and motivation. Section 3 presents the proposed algorithm in detail. Section 4 presents the experimental setting for comparison. Section 5 gives experimental results and a comparison of the algorithm to other algorithms. A further discussion of the algorithms is offered in Section 6. Finally, conclusions are drawn in Section 7.

\section{Background}

\subsection{Dynamic Multi-objective Optimization}

In this paper, we consider that minimization problems and DMOPs [2] [28] can be presented as follows:

$$
\left\{\begin{array}{l}
\min \mathbf{F}(\mathbf{x}, t)=\left(f_{1}(\mathbf{x}, t), f_{2}(\mathbf{x}, t), \ldots f_{m}(\mathbf{x}, t)\right)^{T}, \\
\text { s.t. } g(\mathbf{x}, t) \leq 0, h(\mathbf{x}, t)=0 \\
\mathbf{x} \in[L, U]
\end{array}\right.
$$

where $t$ represents the time variable, and $\mathbf{x}=\left(x_{1}, x_{2}, \ldots, x_{n}\right)^{T}$ is the decision

variable vector. $[\mathrm{L}, \mathrm{U}]=\left\{\mathbf{x}=\left(x_{1}, \ldots, x_{n}\right) \mid l_{i} \leq x_{i} \leq u_{i}, i=1,2, . ., n\right\}$ is the 
decision space, where $\mathrm{L}=\left(l_{1}, \ldots, l_{n}\right)^{T}$ and $\mathrm{U}=\left(u_{1}, \ldots, u_{n}\right)^{T}$ are the lower and upper bounds, respectively. $\mathbf{F}=\left(f_{1}, f_{2}, \ldots, f_{m}\right)^{T}$ is the m-dimensional objective vector and $\mathrm{g}(\mathrm{x}, \mathrm{t}) \leq 0$ and $\mathrm{h}(\mathrm{x}, \mathrm{t})=0$ are the inequality and equality constraints. The definition of DMOPs is a standard formula proposed by Farina et al. [2] 75 and the formula is used in most literature [28] [4]. Thus, constrained DMOPs are not considered in this paper. The time variable, $t$, is associated with the generation number of the EA; $t$ is calculated as follows [2] [5]:

$$
t=\frac{1}{n_{t}}\left\lfloor\frac{\tau}{\tau_{t}}\right\rfloor,
$$

where $\tau$ is the generation number, $n_{t}$ is change severity and $\tau_{t}$ is change frequency.

so Definition 1. Pareto Dominance [21]: Assume that $p$ and $q$ are any two individuals in the population; $p$ is said to dominate $q$, written as $f(p) \prec f(q)$ if $f_{i}(p) \leq f_{i}(q) \forall i \in 1,2, \ldots, m$ and $f_{j}(p)<f_{j}(q) \exists j \in 1,2, \ldots, m$.

Definition 2. Pareto Optimal Set (POS): $x$ is the decision vector; $\Omega$ is the decision space; $F$ is the objective function. A solution is said to be nondominated ${ }_{85}$ if it is not dominated by any other solutions in $\Omega$. Thus, the POS [2] [29] is the set of all nondominated solutions and can be defined mathematically as follows:

$$
P O S:=\left\{x \in \Omega \mid \neg \exists x^{*} \in \Omega, F\left(x^{*}\right) \prec F(x)\right\} .
$$

Definition 3. Pareto Optimal Front (POF): $x$ is the decision vector; $\Omega$ is the decision space; $F$ is the objective function. Thus, the POF is the set of all nondominated solutions with respect to the objective space and can be defined mathematically as follows:

$$
P O F:=\{y=F(x) \mid x \in P O S\} .
$$

Due to the dynamic change of the POS and POF, Farina et al. 2] classified DMOPs into four different types.

- Type I: The POS changes with time but the POF is fixed. 
- Type II: Both the POS and POF change with time.

- Type $I V$ : Both the POS and POF remain fixed.

We mainly deal with the first three types of changes in dynamic multi-objective optimization, although the Type $I V$ change may also occur in some cases.

\subsection{Related Works}

Many DMOEAs have been proposed in recent years and existing approaches [30] [5] can be classified into the following categories: convergence-based methodologies, diversity-based methodologies, and other methodologies [31] [6] according to their ways of managing dynamics [4].

As its name suggests, convergence-based approaches are used to improve the convergence of the population, so as to guide the population to converge to the next POF. Current convergence-based methods mainly include the memory strategy and prediction technique. Memory approaches [7] [32] 33] 34] memorize the previously obtained POS to track the new POS when the environment has regularities. The memory approaches record past historical information to 110 quickly respond to the new environmental change. Peng et al. [34] proposed a memory strategy that preserves some promising solutions. When the environment changes, the method usually selects some nondominated solutions and stores them in a memory pool. Because these elite individuals in memory pool are optimal with best convergence and diversity in past environment. Thus, 115 the approach can increase diversity to some extent. After that, these elite solutions are selected by nondominated selection, and are reused to adapt the new environment. While it is critical to accelerate convergence of the population, for non-periodic problems or the early stages of periodic problems, memory approaches are not as effective as we wish.

Prediction-based mechanisms always apply past population information to forecast some information of the next population and re-initialize the population through certain prediction models. An appropriate prediction model is rather 
essential for the accuracy and effectiveness of the prediction strategy whenever there is an environmental change. Prediction approaches can provide a guiding direction for the evolution of the population towards the POF. In 2006, Hatzakis et al. [32] proposed a feed-forward prediction strategy (FPS). In 2013, Zhou et al. 3] proposed a population prediction strategy (PPS). FPS and PPS use the autoregressive model to predict population, which is effective in solving DMOPs in some degree. However, there are some difficulties for FPS in solving these DMOPs which have a nonlinear correlation between decision variables. This is because FPS only predicts the boundary points of the population, which can not reflect the whole population. In addition, because of the lack of historical information accumulation, PPS has low convergence in the early stage. Muruganantham et al. [35] proposed a prediction model based on the Kalman Filter (MOEA/D-KF). The MOEA/D-KF technique involves a prediction step and a measurement step estimates the current state a priori. In the subsequent measurement, a priori estimate from the MOEA/D-KF is updated to obtain a posteriori. It is applied to the whole population to direct the search towards the next POS instead of the expansion or contraction of the POS or POF manifold. The large prediction errors result in the poor performance of the obtained solutions.

Diversity-based techniques can be classified into two categories according to the period of enhancing the diversity, which are diversity introduction and diversity preservation. Diversity introduction is recognized as the response technique to the environmental changes. Specifically, whenever there is an environmental change, diversity introduction is evoked to generate some diversified solutions to increase the diversity of the population. For example, hyper-mutation methods [36] [8] [37] 38] and random producing solutions [8] 12] 25] are commonly used to help the population escape from the current positions. Additionally, other modified diversity maintenance strategies [39] [40] have been adopted to improve the population's diversity. On the other hand, diversity preservation is not usually designed to explicitly react the changes of environment; instead it mainly focuses on the innate diversity preservation of the optimization algorithms. In 
dCOEA [7], a multipopulation method is proposed to enhance the population diversity in a competitive and cooperative way. In DTAEA [30], a dynamic twoarchive EA is developed to maintain two co-evolving populations, which have complementary effects on enhancing the population diversity. Properly increasing the population's diversity can make the converged population jump out of the current optimum when the environmental change is detected. Appropriate diversity enhancement is essential and rewarding for algorithms to track the changing environment, whereas excessive diversity strengthening may result in low convergence of the algorithms. Based on this idea, many DMOEAs have been proposed, such as co-evolutionary algorithms 7], memetic computing [14], the modeling approach [5] and other methods 38] [41] 29] [42] [43].

165 Aside from the aforementioned approaches, the Particle swarm optimizer (PSO) can deal with MOPs. Proposed by Kennedy et al. [44], PSO is composed of plenty of particles. PSO also has good performance with DMOPs. Helbig et al. 31] introduced a dynamic Vector Evaluation Particle Swarm Optimisation (DVEPSO) algorithm to solve DMOPs. Due to outdated memory and diversity loss, PSO easily moves into local optima when the environmental change is detected. Ant colony optimisation (ACO) is an optimization algorithm based on the natural behavior of ants. It has the ability to deal with multi-objective problems due to its flexibility in being able to add multiple colonies, or multiple pheromone and heuristic matrices. ACO also can be applied to DMOPs because it has the ability to retain useful information when an environmental change occurs. Eaton et al. [45] [46] 47] proposed some approaches based on ACO to cope with a dynamic railway junction rescheduling problem. They found that $\mathrm{ACO}$ has a role to play in a dynamically changing environment and it can deal with real-world dynamic problems.

\subsection{Motivation}

Multi-objective evolutionary algorithm based on decomposition (MOEA/D) [23] is a competent aggregation-based methodology in the community of EMO. Numerous studies have been conducted to improve the quality of MOEA/D in 
static EMO in recent years. Nevertheless, there is little research that relies on improvements of MOEA/D to address DMOPs. Additionally, as already mentioned, current studies on EMO mainly focus on either introducing the diversity to react to environmental changes or maintaining the diversity during the process of optimization. Not many investigations have been explicitly carried out to enhance diversity during the period when searching and reacting occur simultaneously.

In this paper, we put forward a novel Pareto-based evolutionary algorithm using decomposition and truncation (PDTEA) to handle DMOPs. In PDTEA, the decomposition-based algorithm is first adapted to enhance the diversity of the whole population during the optimization process. Then the aggregation functions [23] and Pareto-dominance [21] relationship are used to improve the convergence speed of the population. On the basis of these two steps, a new mating selection strategy, an effective environmental selection technique and a mechanism to handle change in the environment are proposed. The decompositionbased approach is used to select two well-converged and well-diversified mating parents during the mating selection. In addition, an improved truncation operation 22] is adopted during environmental selection for density estimation [22] [48] 49]. If a change is detected, the mechanism to handle changes uses two strategies including an exploration strategy and an exploitation strategy to adapt to the new environment. The first strategy is to explore an individual of the population based on the direction of the individual and its nearest individual which can search for good solutions in the area. The exploitation strategy uses individual variation to enhance convergence. We can use historical information to guide evolution for periodic problems. For each generation, PDTEA select$\mathrm{s}$ the best solutions in each subregion to generate new solutions to accelerate convergence. In the environmental selection, an improved truncation procedure from that of SPEA2 [22] is used to preserve a good distribution of the population for the next generation. 


\section{Proposed Algorithm}

In this section, the decomposition-related preparatory procedure is described 215 first. In order to handle DMOPs, determining how to select efficiently mating parents producing offsprings and preserving good solutions are critical to DMOAs. Thus, a procreation procedure and environmental selection are proposed. Furthermore, a mechanism to handle changes is introduced and we present an overall framework for the proposed algorithm. Finally, we analyze 220 the computational complexity of the compared algorithms and PDTEA.

\subsection{Decomposition-related preparatory procedures}

Given that decomposition-based methodologies are designed to be applied to the evenly distributed reference points in the objective space to ensure diversity before the evolution, a set of reference points $\mathbf{w}=\left(w_{1}, \ldots, w_{M}\right)^{T}$ is produced through a systematic approach [50] [51] [52], where $w_{i} \geq 0$ for all $\mathrm{i} \in\{1, \ldots, M\}$; $M$ is is the number of objectives, and $\sum_{i=1}^{M} w_{i}=1$. The reference points are evenly distributed on an $M-1$-dimension unit simplex, which is a normalized hyperplane. The required generated number of reference points $H$ is closely related to the number of considered divisions along each objective $p$, which can

230 be denoted by the following formula:

$$
H=\left(\begin{array}{c}
M+p-1 \\
p
\end{array}\right)
$$

where $M$ is the number of objectives.

Thereafter, the initialization population $P_{0}$ is normalized in the hyperplane in which the set of reference points is located. First, the ideal point $z_{\min }=$ $\left(z_{\text {min }}^{1}, z_{\text {min }}^{i}, \ldots, z_{\text {min }}^{M}\right)^{T}$ and the worst point $z_{\max }=\left(z_{\text {max }}^{1}, z_{\text {max }}^{i}, \ldots, z_{\text {max }}^{M}\right)^{T}$ of the population $P_{0}$ are calculated respectively, where $z_{\min }^{i}$ and $z_{\max }^{i}$ are the smallest and the biggest value of the i-th objective in the objective space. Thus, the normalized member $\mathrm{x}$ can be calculated as follows [49]:

$$
\hat{f}_{i}(x)=\frac{f_{i}(x)-z_{\min }^{i}}{z_{\max }^{i}-z_{\min }^{i}}
$$


where $\hat{f}_{i}(x)$ denotes the $\mathrm{i}$-th normalized objective of member $\mathrm{x}$. Thus, the objective vector of the normalized individual $\mathrm{x}$ in the population can be denoted ${ }_{240}$ as $\left(\hat{f}_{1}(x), \hat{f}_{i}(x), \ldots, \hat{f}_{M}(x)\right)^{T}$, where $\mathrm{x} \in P_{0}$.

There are several reputable aggregation functions in MOEA/D which convert a multi-objective problem vector into a scalar optimization problem. The first approach is called the Weighted Sum Approach [23]. Its scalar optimization function can be presented as follows:

$$
g(x \mid w)=\sum_{i=1}^{M} w_{i} f_{i}(x)
$$

The solution with the minimization value of $g(x \mid w)$ is regarded as the best solution with the best convergence within the reference point $\mathbf{w}=\left(w_{1}, w_{2}, . ., w_{M}\right)^{T}$. The second approach is the Tchebycheff Approach [23] defined as follows:

$$
g(x \mid w)=\min _{1 \leq i \leq M}\left\{w_{i}\left|f_{i}(x)-z_{\text {min }}^{i}\right|\right\},
$$

The third approach is the Penalty-based Boundary Intersection Approach(PBI) [23], presented as follows:

$$
g\left(x, z_{\min }\right)=d_{1}+\theta d_{2},
$$

$250 \quad$ where $d_{1}=\frac{\left\|\left(f(x)-z_{\text {min }}\right)^{T} w\right\|}{\|w\|}$ and $d_{2}=\left\|f(x)-\left(z_{\text {min }}+d_{1} \frac{w}{\|w\|}\right)\right\|$.

Afterwards, each individual in the population is associated with the reference directions that are generated through reference points and the origin. The reference directions are meant to divide the population into $N$ subregions. For each normalized individual in the population, the perpendicular distance between it and the reference directions is computed. The individual is associated with the reference direction which has the smallest perpendicular distance. This is illustrated in Fig 1, Each individual associated with the reference direction is given an aggregation function value [52] through one specific aggregation function [23].

\subsection{Procreation procedure}

${ }_{260}$ The procreation procedure is adopted to produce offspring individuals from the parent population. The procedure includes two equally critical steps: mating 


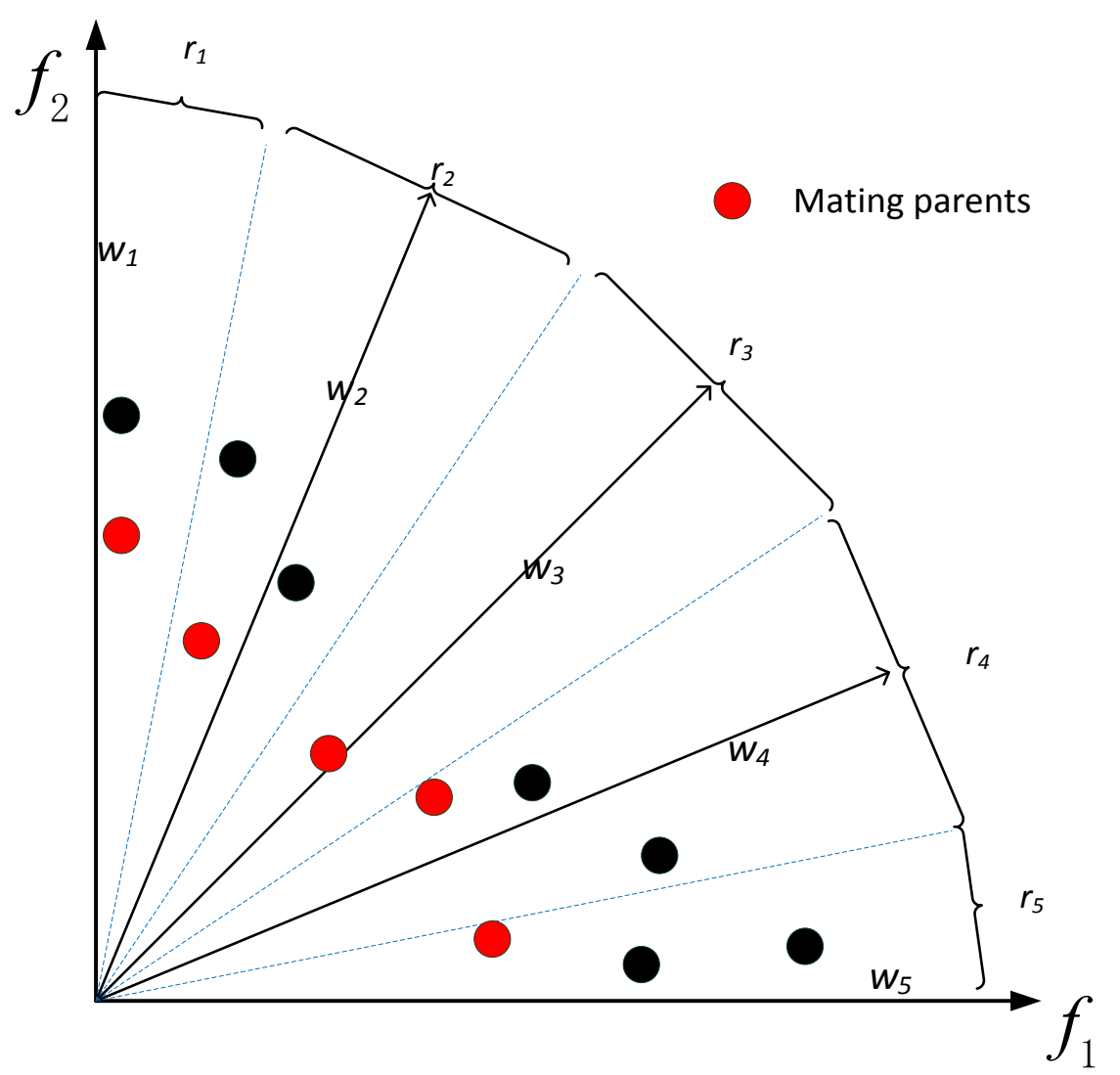

Figure 1: Association of population members and matching selection

selection and generating offspring. The former is to select two mating parents using a specific strategy. Generating offspring, as its name suggests, is used to produce offspring solutions through genetic operators from the chosen mating parents. To enhance the diversity of the population, different from the original MOEA/D, which selects two mating parents from the neighborhood, the proposed mating selection randomly chooses two parents from the $\mathrm{N}$ subregions. After computing the aggregation function values of the individuals associated with the same reference direction, the solution with the best aggregation func270 tion value is selected as one of the mating parents. Another mating parent is chosen in the same way. It can be obtained from Fig 1 that the mating selection 
in PDTEA improves the convergence of the algorithm because individuals with the best aggregation function values are selected as mating parents to produce solutions with good convergence. Considering the association regulation, it is 275 likely that some reference directions have several associated individuals or no individuals. If the chosen subregion does not have associated solutions, it will never be selected. For another situation, when all individuals are only in a subregion, parent individuals are randomly selected by the binary tournament selection [21] from the population.

Then, the offspring generation procedure, using the mating parents to produce the offspring population through the genetic operator, is followed as the mating selection. As for the operator of genetics, in theory, any can be selected to achieve the operator of genetics. In this paper, the simulated binary crossover (SBX) 21] and polynomial mutation (PM) [21] are used as the crossover oper285 ator and mutation operator, respectively. The details of the procreation procedure are shown in Algorithm 1

\subsection{Environmental selection}

The environmental selection is designed to preserve the good solutions of the convergence and diversity performance after the reproduction procedure. The process of environmental selection is presented as follows. The Paretodomination relationship has been proved to be an effective approach to cope with MOPs with two or three goals. Given that most existing DMOPs are problems with no more than three objectives, the non-dominated sort in NSGA-II [21] is first conducted on the combination of the parent and offspring population, 295 after which all the solutions in the union are compared with each other to find the non-dominated levels (i.e., $F_{1}, \ldots, F_{l}, \ldots$, where $l \leq \mathrm{N}$ ), where each solution belongs to 21]. Then, each nondomination level from $F_{1}$ is included in a new population $P$ until the size of $P$ equals to, or first time exceeds the predefined threshold.

In the so-called critical layer $F_{l}$, in order to maintain the diversity of the population, we apply the modified truncation operation changed from that of 


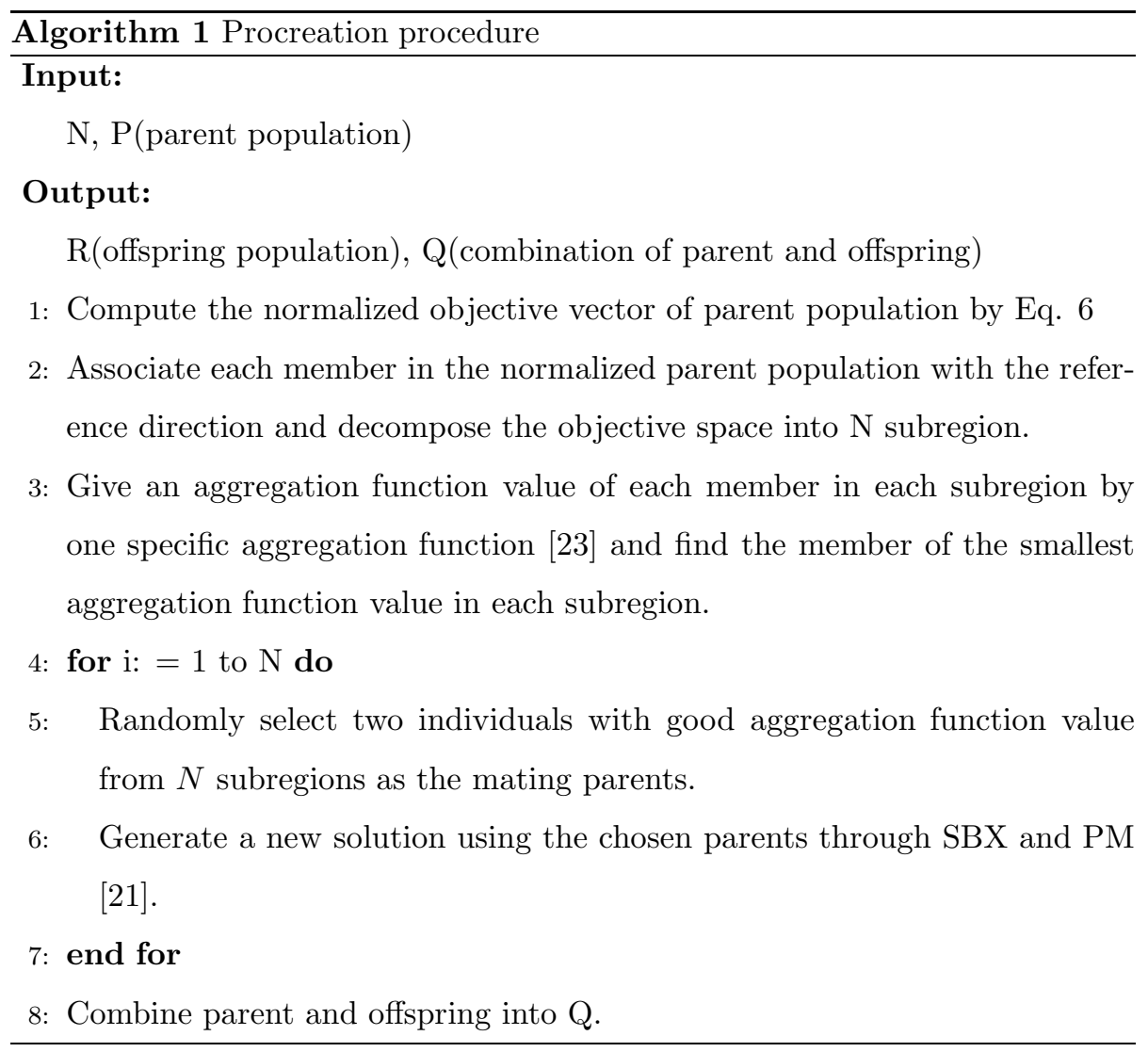

SPEA2 22]. The nearest Euclidean distance of the individuals in the critical layer $F_{l}$ to individuals of selected new population $P$ is computed as follows:

$$
d(q, P)=\min _{p \in P}\|f(q)-f(p)\|
$$

where $q \in F_{l}$. Then, the distance of $F_{l}$ is sorted and the individuals of $F_{l}$ with bigger distance are selected for population $P$. In this way, the whole population's diversity is maintained instead of only in the critical layer. Fig. 2 gives an example to illustrate the reason. Assume that the green points represent the individuals of $F_{1}$, the red and black points belong to the critical layer $F_{2}$. Assume that two individuals of $F_{2}$ need to be selected. In order to improve the whole population's diversity, the red points with the bigger distance are reserved. 


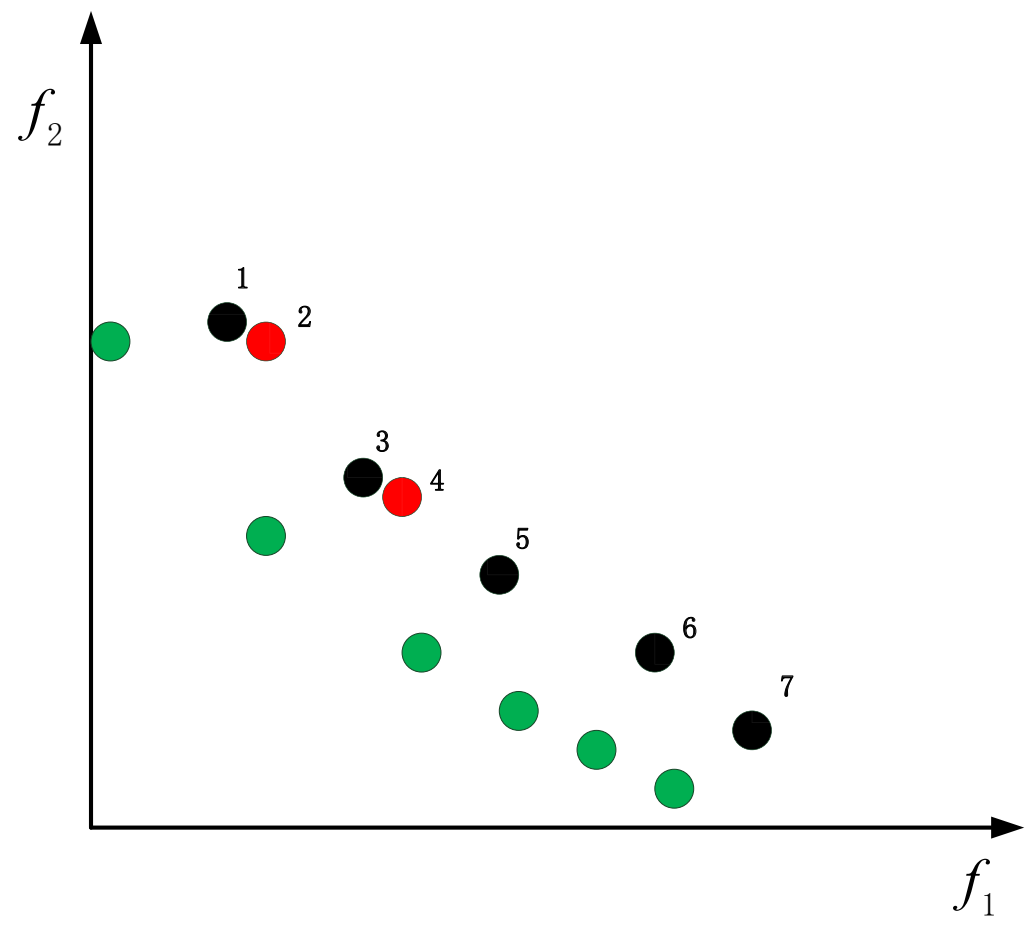

Figure 2: Truncation operation.

\subsection{Dynamic response mechanism}

In order to cope with the two challenges of DMOPs, that is tracing the changing POS and enhancing diversity, in the period of environmental response, this paper proposes a dynamic response strategy based on exploration and exploitation [29] 34]. Exploration guides the whole population to evolve to the region of the next environmental change. Exploitation is applied to adequately search the region that has been located with a local search approach to find more solutions with good convergence and diversity in the decision space.

The exploration strategy is to explore the possible area in which the new population may situate and maintain the diversity of the population to some degree. The direction of individuals can help to guide the convergence of the population and improve diversity in the decision space. Suppose that $x_{t}^{i}=$ 


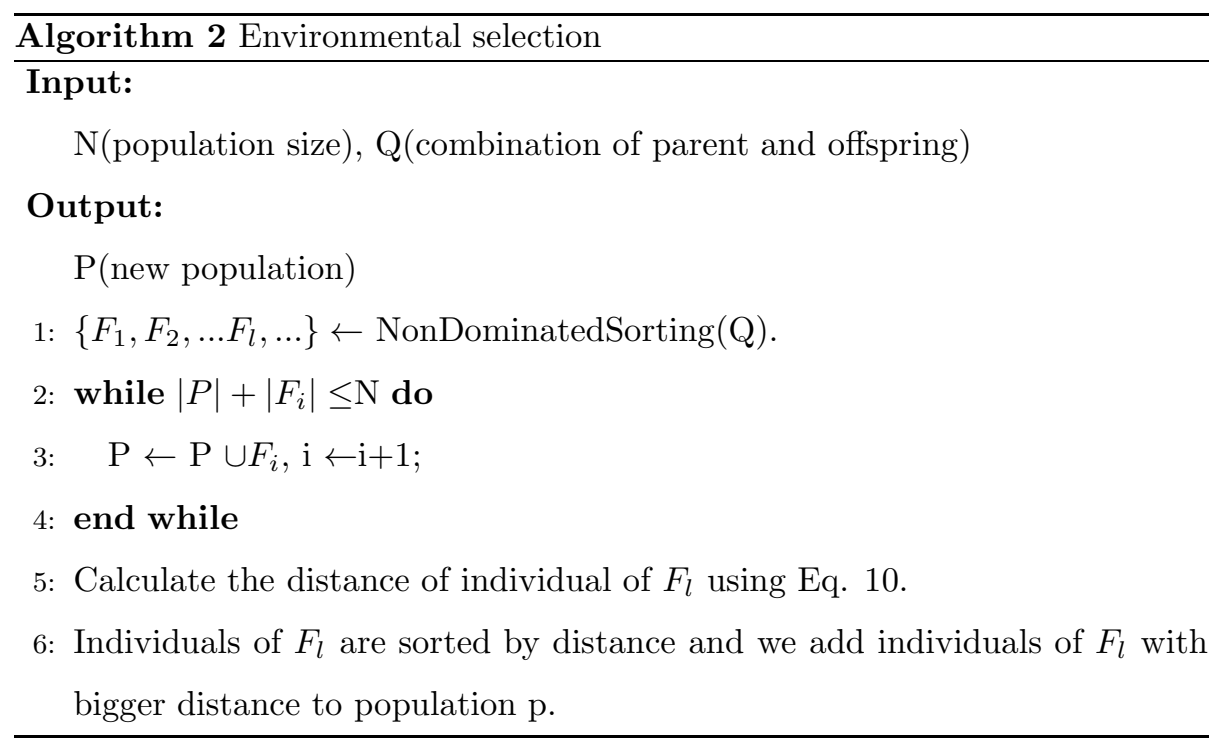

$\left(x_{t}^{i 1}, x_{t}^{i 2}, \ldots, x_{t}^{i n}\right)(i=1,2, \ldots N)$ is the $\mathrm{i}$-th individual population at time $t$, where ${ }_{325} N$ is the population size. For each individual $x_{t}$ in population $t$, there is an individual in population $t-1\left(P_{t-1}\right)$ having the nearest distance to $x_{t}$, which can be found using the following equation:

$$
x_{t-1}^{j}=\arg \min _{y \in P_{t-1}}\left\|y-x_{t}^{i}\right\|_{2}, j=1,2, \ldots, N
$$

where $y$ is an individual of population at time $t$-1. Then, the direction of the $i$-th individual at time $t$ is defined as follows:

$$
D_{t}^{i}=x_{t}^{i}-x_{t-1}^{j}
$$

Then, a variance $\sigma_{t}$ is defined as:

$$
\sigma_{t}=\min _{i=1}^{N}\left\|D_{t}^{i}\right\|, i=(1,2, \ldots N)
$$

where $\left\|D_{t}^{i}\right\|$ is the length of the the i-th individual's direction and $\sigma_{t}$ is the minimum length of the individuals' direction. Then individuals at time $t+1$ are generated by the individuals of time $t$, the moving direction of each individual and the variance according to the following formula:

$$
x_{t+1}^{i}=x_{t}^{i}+D_{t}^{i}+N\left(0, \sigma_{t}\right)
$$




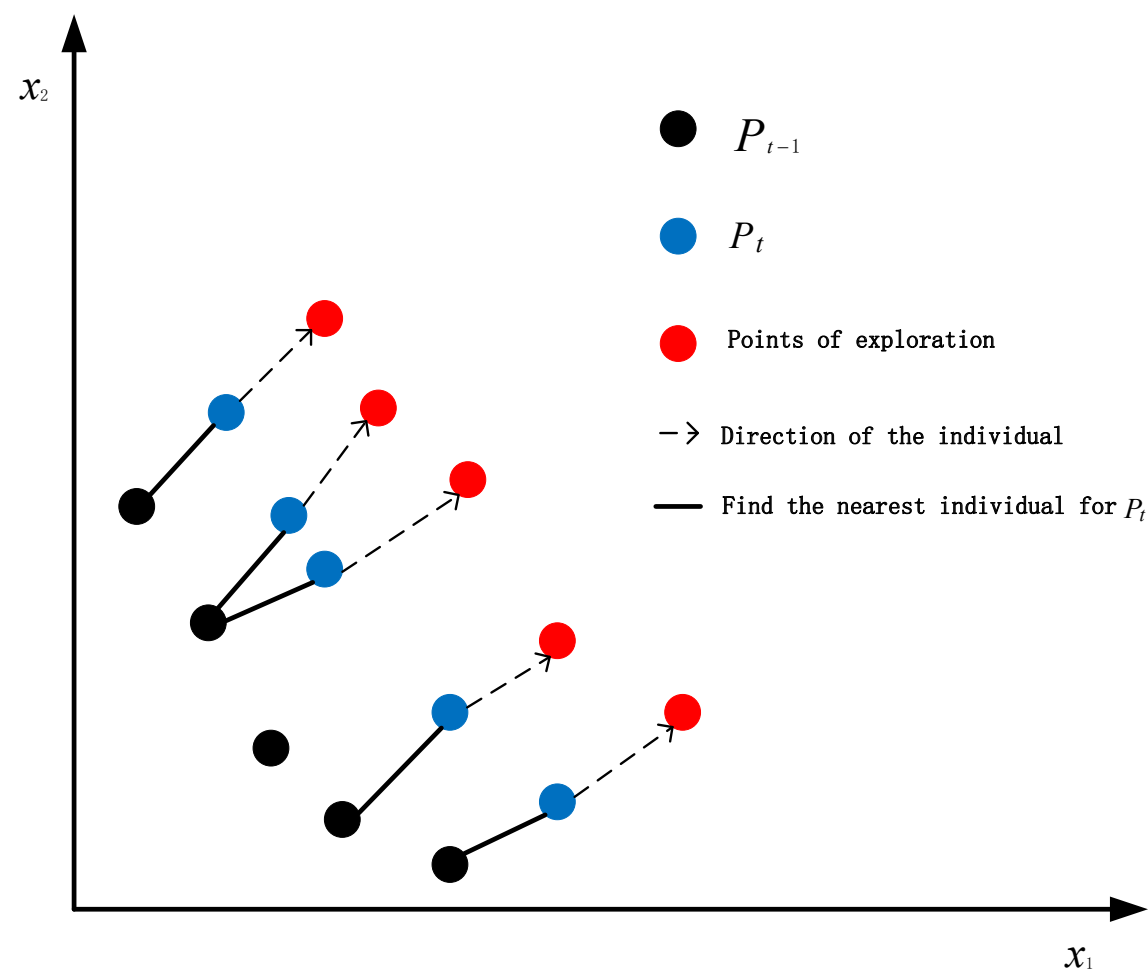

Figure 3: Exploration of the individual.

335 where $N\left(0, \sigma_{t}\right)$ is a random number generated by a Gaussian distribution with a mean of zero and standard deviantion of $\sigma_{t}$. Fig. 3 gives the explanation of how to explore individuals. First, the responding nearest individual of $x_{t}^{i}$ in $P_{t-1}$ is found according to Eq. 11] and then the direction of each individual and the defined variance in terms of Eq. 12 and Eq. 13 are computed. Lastly, $N$ individuals are generated by means of Eq. 14. The main steps of the exploration strategy are described in Algorithm 3 .

After exploring the region of the new POS, another strategy using local search is used to exploit the area around the present POS. First, vector $d_{j}=$ $\left(d_{j}^{1}, d_{j}^{2}, \ldots, d_{j}^{n}\right)$ is defined, then the distances between individual $x_{j}^{i}$ and the low and upper boundary are calculated, and $d_{j i}$ representing the smaller distance is 
denoted as:

$$
d_{j i}=\min \left\{\left|x_{j i}-l_{i}\right|,\left|x_{j i}-u_{i}\right|\right\},
$$

where $j=1,2, \ldots, \mathrm{N} ; i=1,2, \ldots, \mathrm{n} ; l_{i}$ and $u_{i}$ are the low and upper boundaries, respectively. Fig. 4 represents the process of selecting $d_{j}$. Then the $j$-th individual at time $t+1$ is denoted as $x_{t+1}^{j}=\left(x_{t+1}^{j 1}, x_{t+1}^{j 2}, \ldots, x_{t+1}^{j n}\right)$, and $x_{t+1}^{j i}$ can be calculated by the following formula:

$$
x_{t+1}^{j i}=x_{t}^{j i}+N\left(0, d_{j i}\right)
$$

where $x_{i} \in\left[l_{i}, u_{i}\right], \mathrm{d}=\left(d_{1}, d_{2}, \ldots d_{n}\right), n$ is the dimension of the decision space and $d_{j i}$ is the variance of the Gaussian white noise. The process of exploiting is illustrated in Fig. 4. The steps of the exploitation strategy are presented in Algorithm 3 .

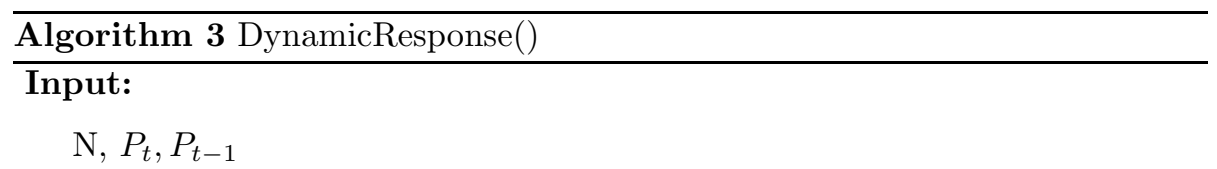

\section{Output:}

$P_{\text {exploration }}, P_{\text {exploitation }}, \mathrm{P}$ (parent population);

1: Find the individual in $P_{t-1}, x_{t-1}^{j}$ closest to the $x_{t}^{i}$ using Eq. 11.

2: Calculate $D_{t}$ and the variance $\sigma_{t}$ using Eq. 12 and Eq. 13 , respectively.

3: Generate $\mathrm{N}$ individuals according to Eq. 14 as $P_{\text {exploration }}$

4: Calculate $d_{j}$ using Eq. 15

5: Generate $\mathrm{N}$ individuals according to Eq. 16] as $P_{\text {exploitation }}$.

6: Combine the two obtained populations and set the combined population as $P_{\text {combine }}, P_{\text {combine }}=P_{\text {exploration }} \cup P_{\text {exploitation }}$.

7: Select $\mathrm{N}$ individuals from $P_{\text {combine }}$ by Algorithm 2 and set the population as $\mathrm{P}$.

It should be noted that the proposed change response method is different from prediction approaches. Prediction approaches need past historical information to predict the next population. Hence, the proposed approach first uses exploration-based strategy to guide the whole population toward the promising 


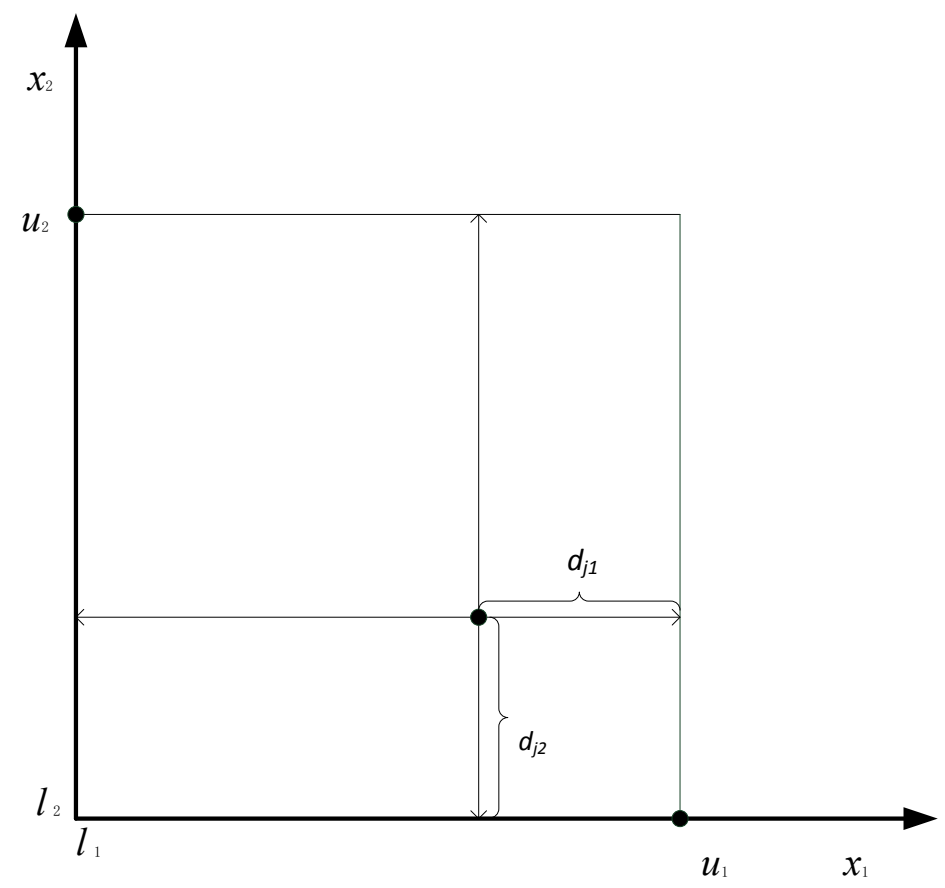

Figure 4: local search of individual.

region's evolution. The exploration strategy can generate some solutions close to the new POS to improve convergence of the population. Moreover, to achieve the exploration, the exploitation strategy is employed to search for some preferable individuals using local search when the environment changes. Hence, the strategy based on exploration and exploitation can benefit the population to adapt to the new environment quickly.

\subsection{Overall framework of the proposed algorithm}

The overall framework of PDTEA is proposed in Algorithm 4. First, the initial procedure produces the initializing population $P_{0}$, time $t=0$ and iteration generation gen. Afterwards, in every iteration, environmental changes are detected. If there is a change, the response mechanism is invoked. Otherwise, 
370 the optimization process is imposed on the whole population during which the reproduction procedure and environmental selection are adopted to produce the next generation's initialized population. Within the optimization process, the reproduction procedure is used to generate offspring individuals. Then, environmental selection is exerted on the set including the parent population and the offspring. In the following subsections, the detailed implementation of each component in PDTEA is exhibited step by step.

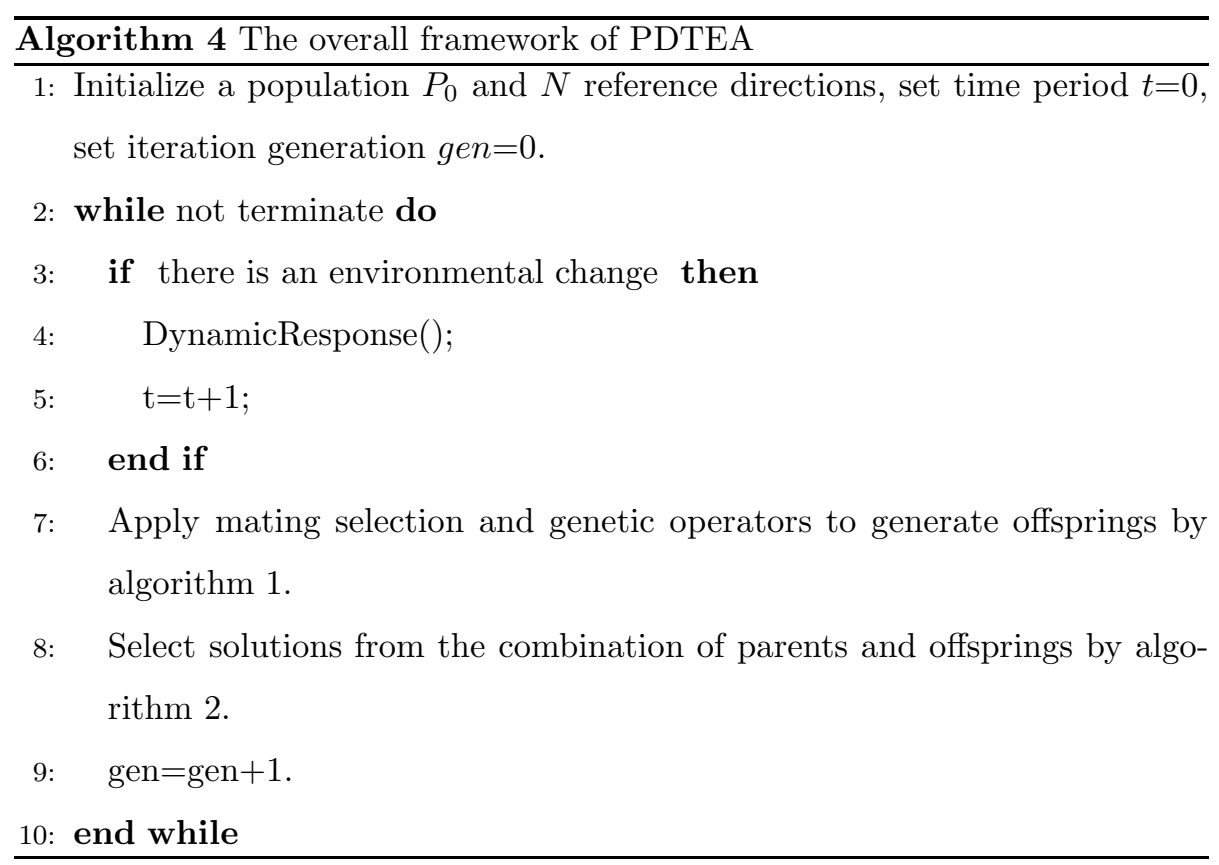

\subsection{Computational complexity of the compared algorithms and PDTEA}

The optimization algorithm consumes the most computational resources of the compared algorithms and PDTEA. The computational complexity of each 380 optimization algorithm and PDTEA are analyzed as follows:

(1) DNSGA- $I I$ : From the original paper of DNSGA- $I I$ [9], the optimization algorithm is NSGA-II [21] and the computational resource is spent on nondominated sorting $\mathrm{O}\left(M(2 N)^{2}\right)$, crowding-distance assignment $\mathrm{O}(M(2 N) \log (2 N))$ 
and sorting $\mathrm{O}(2 N \log (2 N))$. The overall computational complexity is $\mathrm{O}\left(M N^{2}\right)$, $\mathrm{M}$ is the number of objectives and $\mathrm{N}$ is the population size.

(2) PPS: PPS [3] chooses RM-MEDA [53] as the MOEA optimizer. In RMMEDA, the computational complexity of RM-MEDA includes modeling, reproduction and the selection operator. The modeling cost is $\mathrm{O}(\mathrm{nN}) ; \mathrm{n}$ is the number of the decision space. The reproduction spends $\mathrm{O}(\mathrm{nK}) ; \mathrm{K}$ is the number of clusters. The selection operation is NSGA-II 21]. Therefore, the overall computational complexity is $\mathrm{O}\left(M N^{2}\right)$.

(3) MOEA/D: As introduced in Section 4.2 the computational complexity mainly depends on updating neighboring solutions. It costs $\mathrm{O}(\mathrm{MNT})$ computations; $\mathrm{N}$ is the population size, and $\mathrm{T}$ is the number of subproblems. Therefore, the overall computational complexity is $\mathrm{O}(\mathrm{MNT})$.

(4) SGEA: SGEA [4] is introduced in section 4.2] and it consumes during steadystate evolution and environmental selection. The whole steady-state evolution part takes $\mathrm{O}\left(M N^{2}\right)$ computations and the environmental selection procedure spends $\mathrm{O}\left(M N^{2}\right)$ computations. Therefore, the overall computational complexity is $\mathrm{O}\left(M N^{2}\right)$.

(5) Dy-NSGA-II: Dy-NSGA-II 6] adopts NSGA- $I I$ as the optimization algorithm. The computational complexity of NSGA-II has been analyzed and it is $\mathrm{O}\left(M N^{2}\right)$.

(6) PDTEA: For the overall framework of each generation, the main computational resource in PDTEA is consumed by environmental selection and the offspring reproduction. Two strategies also need computational resources when an environmental change is detected. Identifying the ideal point and worst point requires a total of $\mathrm{O}(\mathrm{MN})$ computations, and association of population members to $\mathrm{H}$ reference points requires $\mathrm{O}(\mathrm{MNH})$ computations. The offspring reproduction (line 7 of Algorithm 4) requests $\mathrm{O}\left(M N^{2}\right)$, where $\mathrm{M}$ is the number of objectives and $\mathrm{N}$ is the population size. The computational complexity of environmental selection (line 8 of Algorithm (4) is $\mathrm{O}\left(M N^{2}\right)$. Therefore, the overall computational complexity of PDTEA in each generation is $\mathrm{O}\left(M N^{2}\right)$. 
Due to these analyses, the computational complexity of PDTEA is similar to the compared algorithms except MOEA/D.

\section{Experimental Design}

In this section, we introduce test problems, the compared algorithms, performance metrics and parameter settings.

\subsection{Test Problems}

Twenty-one dynamic multi-objective test instances (FDA1-5, dMOP1-3, $J Y 1-9, d M O P 2_{i s o}, d M O P 2_{d e c}, F D A 5_{i s o}$ and $\left.F D A 5_{d e c}\right)$ were used to assess our algorithm. Farina et al. [2] proposed the FDA test suite and Goh et al. 7] proposed the dMOP test suite. FDA and dMOP have linear correlations 425 between decision variables and are widely used to assess the performance of DMOEAs [3] 4]. However, the POS of real world problems is not so simple. The JY test suite, which has a linear correlation between the decision variables, was proposed by Jiang et al. [28], some of which has nonlinear correlation between the decision variables. It introduced characteristics, such as mixed POFs and a nonmonotonic and time-varying relationship between variables, which are very competent and beneficial when testing the performance of algorithms. Helbig et al. 54]55] proposed some new DMOPs with a complicated POS, and dynamic multi-objective benchmark functions were selected to assess the performance of the algorithm.

\subsection{Compared Algorithms}

In this section, the proposed algorithm is compared with six popular DMOEAs. They are the MOEA based on decomposition (MOEA/D) [23], the dynamic version of NSGA-II (DNSGA- $I I)$ 9], the population prediction strategy (PPS) 3], a steady-state and generational evolutionary algorithm (SGEA) 4], ${ }_{440}$ a dynamic version of the Non dominated Sorting Genetic Algorithm II(DyNSGA-II) 6] and the dynamic vector evaluation particle swarm optimization (DVEPSO) 31] . A brief description of each compared algorithm follows: 
(1) DNSGA-II: NSGA-II [21] is a classical algorithm based on Paretodominance. In order to adapt to dynamic optimization problems, Deb et al. 9] modified the commonly utilized NSGA-II to track the POF. Some population members are replaced with either randomly produced solutions or mutated solutions of existing population solutions when a change occurs.

(2)PPS: PPS predicts a whole population rather than isolated points. In PPS[3], the POS is divided into two parts: the population and manifold. PPS chooses a univariate autoregression model to predict the next population center by the archived population centers over a number of continuous time series. Similarly, previous manifolds are used to predict the next manifold. The initial population is initialized by the predicted center and manifold when an environmental change occurs.

(3)MOEA/D: MOEA/D provides an efficient way to optimize MOPs. MOEA/D can decompose a multi-objective optimization problem into a number of scalar optimization subproblems and optimize them simultaneously[23]. Each subproblem is optimized from information of its several neighboring subproblems. The neighborhood of subproblems is composed through the distances between 460 their aggregation coefficient vectors. The diversity of population is controlled by the diversity of subproblems and the convergence of the population is vulnerable to the neighborhood of each subproblem and solution update in this neighborhood.

(4)SGEA: SGEA can make use of the fast and steady tracking ability of 465 steady-state algorithms and the good diversity preservation of generational algorithms for solving DMOPs. Mating selection parents are selected either from the parents' population or the archive population, and environmental selection preserves good solutions for improving the convergence speed of the population. Some old solutions with good diversity are reused and information from the previous environment and new environment are used for reacting to environmental changes when a change is detected.

(5)Dy-NSGA-II: Azzouz et al. [6] proposed a new dynamic NSGA-II(DyNSGA- $I I$ ) based on an adaptive hybrid population management strategy in- 
cluding memory, local search and random strategies. The local search approach 475 is used to guide the population towards the promising regions according to finding direction in search space. The memory approach is used to store former information of the POS that is exploited to help the population quickly track the POS when the change degree is small. The role of the random approach is to deal with environmental change having a large severity.

(6) DVEPSO: DVEPSO was proposed by Helbig et al. [31] to solve DMOPs. DVEPSO was inspired by the vector evaluation particle swarm optimization algorithm. It uses various ways to manage the archive solutions and knowledge sharing through local and global update approach for the search process. When an environmental change occurs, a percentage of the swarm's particles ${ }_{485}$ are reinitialized and all particles' pbest and the swarm's gbest are reevaluated.

\subsection{Performance Metrics}

In this section, performance metrics, which can evaluate convergence, distribution and diversity of the obtained solution set, are introduced.

1)Generational Distance (GD): Veldhuizen et al. 77] 34] presented the GD 490 metric, which measures the convergence of the population. The GD indicator is defined as follows:

$$
G D\left(P O F_{t}, P_{t}\right)=\frac{\sum_{v \in P_{t}} d\left(P O F_{t}, v\right)}{\left|P_{t}\right|},
$$

where $d\left(P O F_{t}, v\right)=\min _{u \in P O F_{t}} \sqrt{\sum_{j=1}^{m}\left(f_{j}^{v}-f_{j}^{u}\right)^{2}}$ is the minimum Euclidian distance between $v$ and the point in $P O F_{t}$. $P O F_{t}$ is a set of uniformly distributed Pareto optimal points in the POF at time t; $P_{t}$ is the solution obtained 495 by the algorithms.

2)Inverted Generational Distance(IGD): IGD [3] [29] is a metric, which assesses the convergence and diversity of the obtained solution set. The IGD is calculated as follows:

$$
I G D\left(P O F_{t}, P_{t}\right)=\frac{\sum_{v \in P O F_{t}} d\left(v, P_{t}\right)}{\left|P O F_{t}\right|},
$$


where $d\left(v, P_{t}\right)=\min _{u \in P_{t}} \sqrt{\sum_{j=1}^{m}\left(f_{j}^{v}-f_{j}^{u}\right)^{2}}$ is the minimum Euclidian distance 500 between $v$ and the point in $P_{t}$. $P O F_{t}$ is a set of uniformly distributed Pareto optimal points in the POF at time $t ; P_{t}$ is the solution obtained by the algorithms. The IGD [3] performance metric is a comprehensive index and is developed to measure the convergence and diversity of the algorithm' obtained solutions.

3) Hypervolume Difference(HVD): The HVD [8] [4] [56] measures the gap between the hypervolume of the obtained POF and that of the true POF.

$$
H V D\left(P O F_{t}, P_{t}\right)=H V\left(P O F_{t}\right)-H V\left(P_{t}\right)
$$

where $P_{t}$ is the solution obtained by the algorithm at time $\mathrm{t}$ and $P O F_{t}$ is the solution of the true POF at $t$ time. $H V(S)$ is the hypervolume of a set $S$. The reference point for the computation of hypervolume is $\left(z_{1}^{t}+0.5, z_{2}^{t}+0.5, \ldots, z_{M}^{t}+\right.$ 0.5 ), where $z_{j}^{t}$ is the maximum value of the $j$ th objective of the true POF at $t$ ${ }_{510}$ time and $M$ is the number of objectives.

\subsection{Parameter Settings}

The experimental parameters were set as follows. The population size was $\mathrm{N}=100$. The dimensions of the test problem's decision space were $n=20$. For change detection, $5 \%$ of the population was randomly selected and re-evaluated to detect environmental changes. It should be noted that re-evaluated approaches for change detection assume there is no noise in function evaluations. Each algorithm ran independently 20 times on all problems, and there were 120 environmental changes. Due to its selection in many papers [57] [58], the PBI method is employed in this paper and we set $\theta=5.0$. The Wilcoxon rank-sum test [59] was used to point out significance between different results at the 0.05 significance level. The parameters of the MOEAs compared algorithms were referenced from their original papers. Some key parameter settings in the papers were listed as follows:

1)MOEA/D: The size of subproblems was set to 100. In order to deal with FDA4 and FDA5, 1000 weight vectors were generated by the simplex-lattice design [51]; these were reduced to 100 using SPEA2 [22]. The neighborhood size 
and the maximal number of solutions that could be replaced were set to 20 and 2 , respectively. Additionally, the aggregation function used in the experiment was the PBI method, where $\theta=5.0$.

2) For all algorithms, the crossover probability was $p_{c}=0.8$ and its distribution index was $\eta=20$. The mutation probability was $p_{m}=1 / \mathrm{n}$ and its distribution $\eta=20$.

We did not tune the parameters one by one to get better experimental results. If some parameters in algorithms are adjusted separately, we can get better 535 experimental results. Therefore, the parameter settings of all algorithms were the same to ensure that the comparisons were fair.

\section{Experimental Results and Analysis}

In order to compare the effect of change frequency on the compared algorithms in dynamic environments, the severity of change was fixed to 10 , and the frequency of change was set to 20,25 and 30 , respectively. The statistical results of seven algorithms and the mean and standard deviation values of GD, IGD and HV are shown in Table 1, Table 2 and Table 3, respectively. The best values obtained by the seven algorithms are highlighted in bold face, and the Wilcoxon rank-sum [59] test was carried out to indicate significance between different results at the 0.05 significance level.

\subsection{Results on FDA and dMOP problems}

It can be obtained from Table 1 that PDTEA has the minimum values of GD on the majority of FDA and dMOP test suites whose decision variables are linearly related. The smaller values of GD imply that the algorithm had better convergence than the other algorithms. On the whole, PDTEA significantly shows the best convergence among all the compared algorithms on most test problems. For all the problems, at whatever the frequency of change was set, PDTEA significantly performed better than DNSGA-II, PPS, Dy-NSGA-II and DVEPSO. PDTEA surpassed MOEA/D on all FDA and dMOP problems 
except FDA2 and dMOP1. However, when compared with SGEA, PDTEA failed to show better competition on FDA2, FDA3 and dMOP1. The reason is that the POS of FDA2 and dMOP1 remain fixed. MOEA/D and SGEA preserve many solutions from the last population, which has considerable convergence merits when addressing those DMOPs with unchanged POS.

As shown in Table 2. PDTEA's IGD performance metric was the best in most of the test problems except FDA2, FDA3 and dMOP1. Therefore, not only did PDTEA have better distribution than the other methods, but also significantly surpassed others in terms of convergence. For FDA2 and FDA3 test instances, the IGD values of SGEA were the best and those of PDTEA ranked the second, proving that the distribution and convergence of PDTEA were only weaker than SGEA in dynamic changes. As for dMOP1, the values of IGD on MOEA/D were the smallest, which were smaller than SGEA and PDTEA. The conclusion can be made that PDTEA performs moderately on problems like dMOP1.

The HVD values were roughly similar to the IGD values on FDA and dMOP displayed in Table 2 and Table 3. The difference is that the number of HVD values on which PDTEA performed best is one more than that of the IGD values. Specifically, for FDA3, PDTEA significantly outperformed all the other approaches in terms of the HVD metric. In addition, PDTEA ranked the second on problem dMOP1 rather than the third, which can be seen in Table 2 Perhaps the main reasons for the analogous performance are they are comprehensive metrics that measure both distribution and convergence. Obviously, PDTEA is preferable to the other algorithms on most FDA and dMOP problems. However, it is slightly inferior to SGEA on FDA2 and dMOP1 indicating SGEA is also 580 promising as a means to solving DMOPs. It should be mentioned that PDTEA showed significantly competent performance on FDA4 and FDA5 in terms of GD, IGD and HVD values, indicating that PDTEA is the most effective and outstanding methodology for solving problems with three goals. 
Table 1: Mean and SD of GD indicator obtained by seven algorithms.

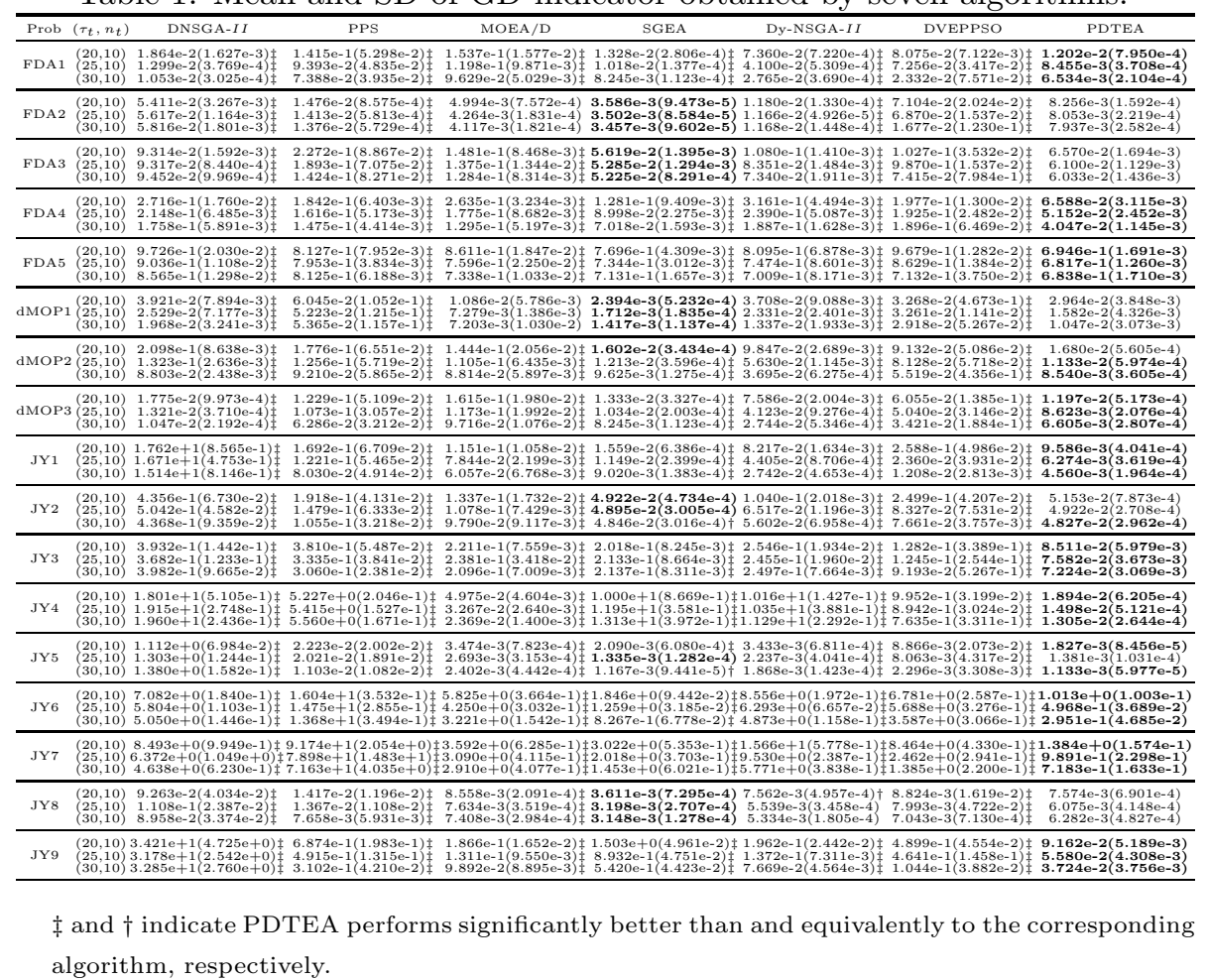

\subsection{Results on JY problems}

Compared with FDA and dMOP problems, JY problems [28] are a new benchmark suite with several complex characteristics including a nonmonotonic and time-varying relationship among decision variables. Apart from that, the changing types of some problems vary with time from one to another during the optimization process. It can be obtained from Table1 that PDTEA significantly performed best over other approaches on most JY problems except JY2, JY5 and JY8. As for the JY2 problem, PDTEA only showed less convergence than SGEA but obvious significance than the other five methods. In addition, as changing frequency increased, the superiority of SGEA was not so significant. It is likely that PDTEA would outperform SGEA if the frequency of change were to increase to some certain value. When it comes to JY5, PDTEA almost performed as well as SGEA. It might be concluded that the less the changing 
frequency, the better PDTEA performs. For JY8, whose geometry and the number of mixed segments of the POF vary over time, PDTEA performed better than SGEA, which suggests that the decomposition-based mating selection of PDTEA may have had a negative effect on the convergence.

It can be seen from Table 2 that the IGD values of PDTEA were smallest on almost all tested problems except JY3, JY5 and JY8, indicating that PDTEA showed almost best diversity and convergence on almost all of the DMOPs apart from JY3, JY5 and JY8. PDTEA only showed worse performance than SGEA on JY5. Additionally, the HVD values of all the compared algorithms can be found in Table. 3. Obviously, the HVD values of PDTEA only performed worse than the other methods on JY5 and JY8, which shows that the PDTEA's comprehensive performance in terms of diversity and convergence was only worse when solving problems like JY5 and JY8. All algorithms' values of HVD on JY5 were the same as those of IGD. It should be noted that PDTEA performed less effectively on JY8, since it only outperformed DNSGA-II, and it performed equally to PPS. However, it also showed worse performance when compared with SGEA. To conclude, the diversity and convergence of PDTEA is worse than that of SGEA on JY5 and JY8. The reason might be that both JY5 and ${ }_{615}$ JY8 have the fixed POS, suggesting that PDTEA has less competent performance when solving DMOPs with an unchanged POS. Additionally, PDTEA demonstrated more significant effectiveness and superiority than most existing DMOEAs when addressing problems with considerable complicated geometry and rather sophisticated characteristics.

\subsection{Results on $F D A 5_{i s o}, F D A 5_{d e c}, d M O P 2_{\text {iso }}$ and $d M O P 2_{\text {dec }}$}

The flat regions and a deceptive POF were proposed by Huband et al.[60] and Helbig et al. [55] [54] introduced some dynamic problems with new dynamic features. We selected $F D A 5_{i s o}, F D A 5_{d e c}, d M O P 2_{i s o}$ and $d M O P 2_{d e c}$ to compare the algorithms with $\left(\tau_{t}, n_{t}\right)=(25,10)$ and obtained GD, IGD and HVD 625 metric values in Table 4. The Wilcoxon signed-rank test [59] was carried out at the 0.05 significance level to indicate statistically significant differences between 
Table 2: Mean and SD of IGD indicator obtained by seven algorithms.

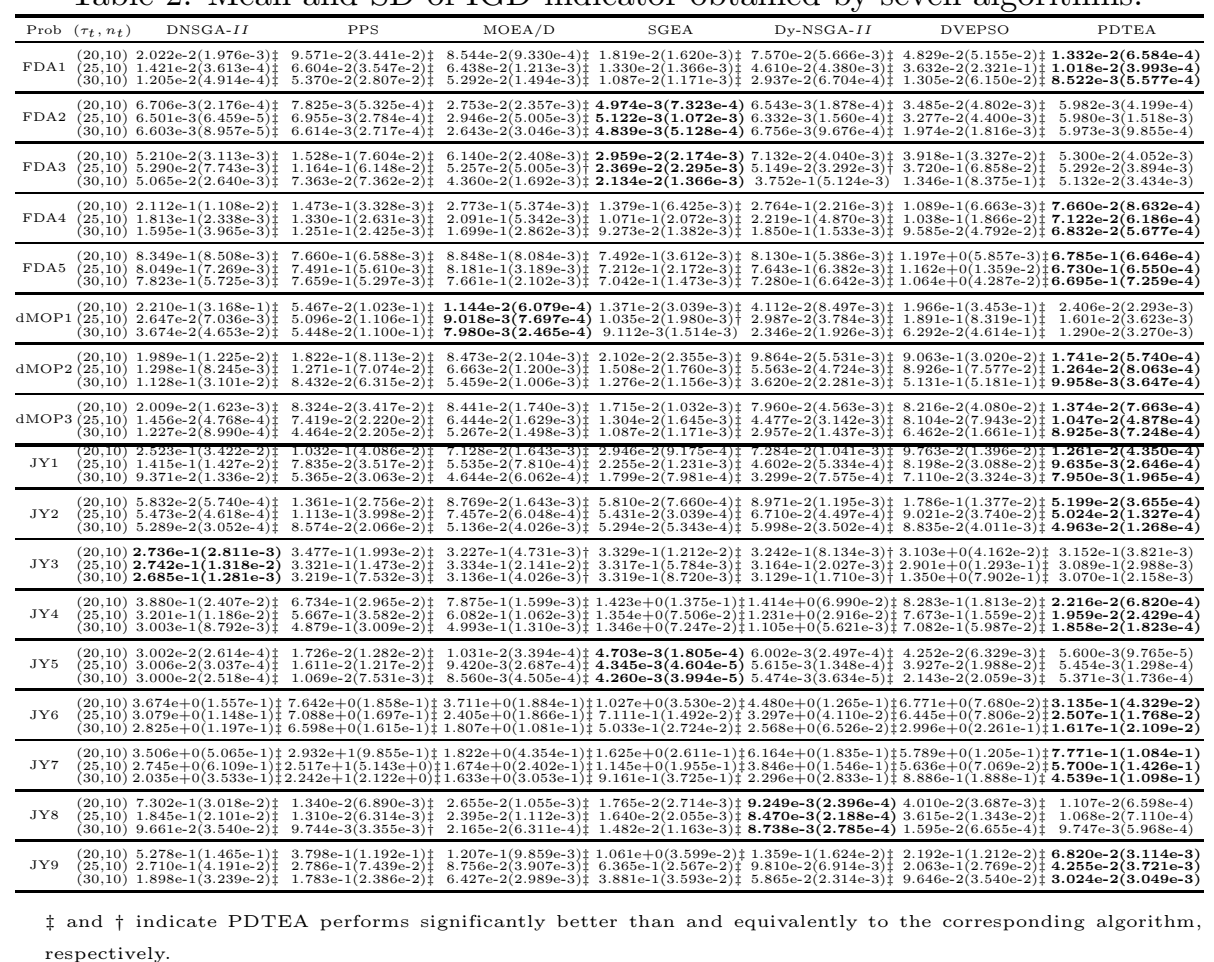

DPTEA and the other algorithms.

It can be observed from Table 4 that most algorithms obtained worse metric values on the problems with dynamic features, implying the problem is challenging for DMOEAs. PDTEA can deal with a majority of problems except $d M O P 2_{i s o}$ according to three metrics. The result of SGEA on $d M O P 2_{i s o}$ indicates that SGEA is a top performer on the problem. This is because SGEA uses the steady-state population update to significantly improve performance. For $F D A 5_{d e c}$ with a deceptive POF, PDTEA shows superior performance by IGD and HVD values, while the GD value indicates SGEA has a good convergence performance. The reason is that PDTEA uses the exploration strategy to improve the population's diversity. Hence, the exploration strategy is helpful to deal with the deceptive POF. Nevertheless, the steady-state update in SGEA may cause the loss of diversity. 
Table 3: Mean and SD of HVD indicator obtained by seven algorithms.

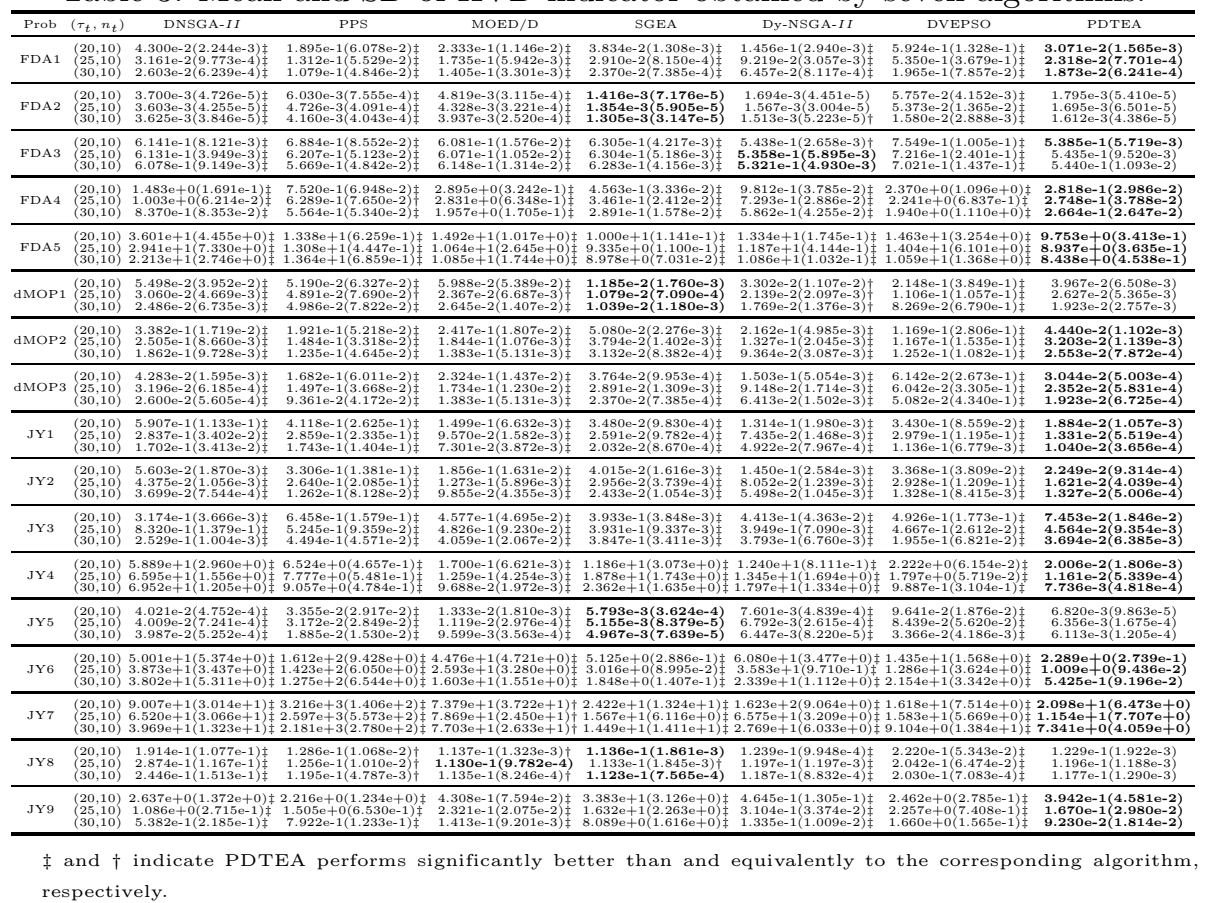

Table 4: Mean and SD of three indicators obtained by seven algorithms.

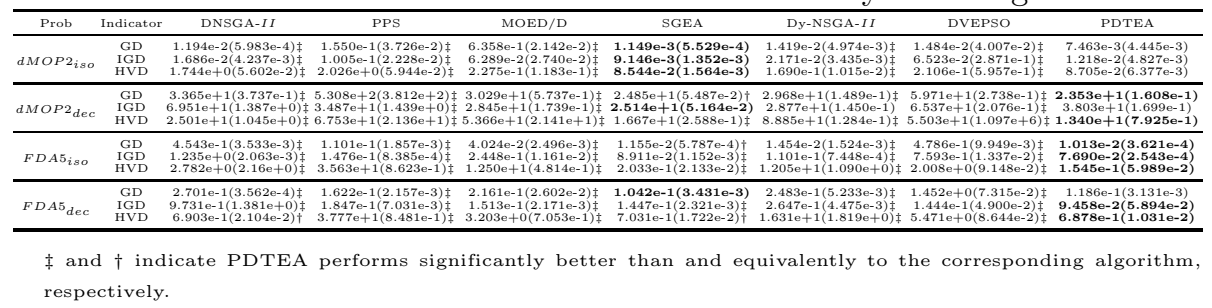

\subsection{Comparing evolutionary processes of different algorithms}

In order to clearly compare the algorithms' performance, evolution curves of the average IGD values are drawn up in Fig. 5. It can be observed that PDTEA could quickly respond to environmental changes on most of the test problems. For FDA1, dMOP2 and dMOP3, the IGD values of PDTEA are relatively smaller than others in the early stage before the 30th environmental change, after which the IGD values of PDTEA are bigger than that of PPS. The reason might be that PPS requires considerable accumulation of historical 
information to respond to environmental changes. Additionally, PPS can make the best use of the accumulated historical information to accurately predict the whole population, thereby performing best at the latter stages. For FDA4 and FDA5, the evolution curve of PDTEA's IGD values is almost the lowest in the whole stage of evaluation, proving that PDTEA significantly performed better than the others. One possible reason is that decomposition-based mating selection can assist the optimization when dealing with three-objective dynamic problems. However, it can be seen from the indicator's evolution curves of FDA2 and dMOP1 that PDTEA showed slightly overall worse performance than SGEA. Since SGEA uses more past solutions, including half of old solutions, to cope with the dynamics, it is superior to the others when addressing problems with fixed POS. SGEA can quickly approximate to the new POS when the environmental change comes. Lastly, for FDA3, PDTEA and SGEA showed best performance in turn every 20 environmental changes, which can be found in Fig. 5 (c). Because the density of solution on POF can vary from time and the population can not have good distribution.

It is clear from Fig. 6 that PDTEA had the best performance on JY4, JY6 and JY7 among all the compared algorithms. Given that the POF of JY4 is discontinuous and JY6-JY7 are multimodal problems, PDTEA is very suitable and outstanding for addressing those problems with discontinuous POF and multimodality. For JY1 and JY5, all the algorithms's performances were similar to those of FDA1 and dMOP1, respectively. For JY2 and JY8, PDTEA and PPS had slightly better performance than the other approaches in turn, which can be obtained from Fig. 6 (b)(h). This implies that PDTEA and PPS have relatively but not very significantly better performance when solving DMOPs whose varying POFs have geometry of mixed segments or oscillate among several modes with the changing environment. For JY3 with a time-varying and nonmonotonic relationship between variables, all the compared approaches do not show encouraging results and their performances did not have any statistical regularity. Therefore, most existing algorithms must be greatly improved to address these kinds of problems, which is one of our future goals. Lastly, for 
JY9, which had a mixed changing type and a dramatic environmental change ${ }_{680}$ during the evolution, PDTEA had better steady performance on JY9, suggesting it can handle geometry of mixed segments. Lastly, for JY9, PDTEA could tackle the problem, performing better than the other methods except for the period of dramatic change, which indicates that PDTEA is excellent for solving the problem with varying types of changes, but is less effective when dealing with dramatic changes that occur during optimization.

\subsection{Comparison of the distribution of the final obtained population}

In order to compare the performance of the final obtained population, we selected FDA1, dMOP2, JY2 and JY6 to draw the final population distribution of seven algorithms. Six moments were selected to analyze results on FDA1, JY2 and JY6 as shown in Fig. 7, Fig. 9 and Fig. 10. Eight moments were selected in Fig. 8. The figures indicate that PDTEA had better convergence and distribution in the early stages, implying it can quickly respond to environmental changes. The reason might be that the exploitation and exploration in the dynamic response strategy can help the population to respond to the environmental changes accurately and quickly. It is obvious that PPS is worse than the other algorithms because historical information stored by PPS at the early stages is very small. For multimodal problem JY6, PDTEA performed better than the other algorithms which can be seen from Fig 10. Thus, when dealing with difficult problems, PDTEA has a significant advantage over the other algorithms, especially in the speed at which it can respond to environmental changes.

\section{Discussion}

In the section, we discuss the influence of change, different components of PDTEA, different dimensions of the decision space and the statistical analysis. 


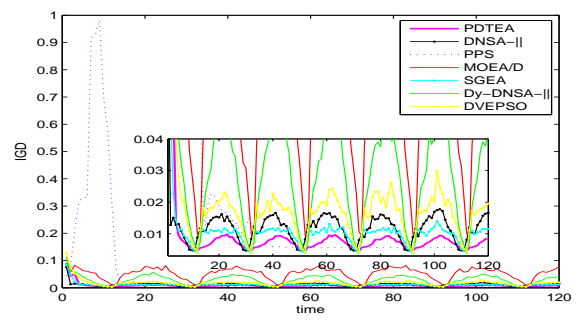

(a) FDA1

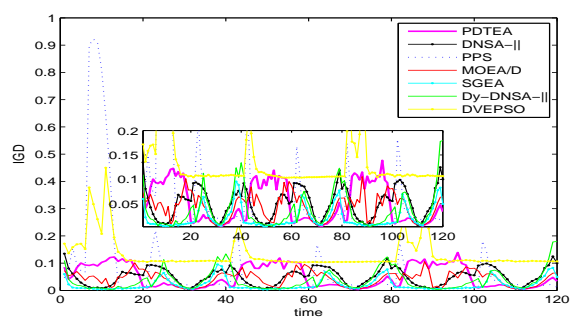

(c) FDA3

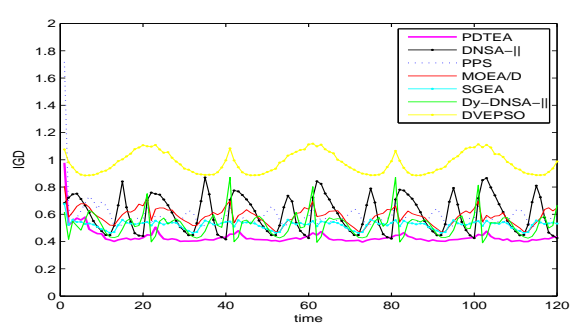

(e) FDA5

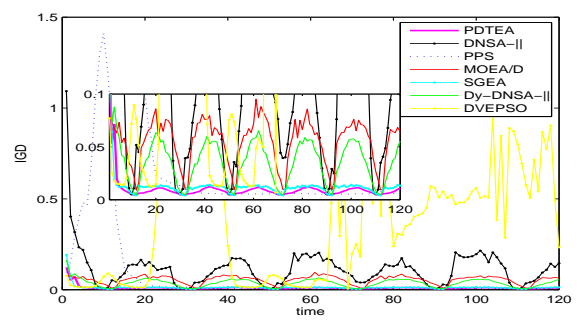

(g) dMOP2

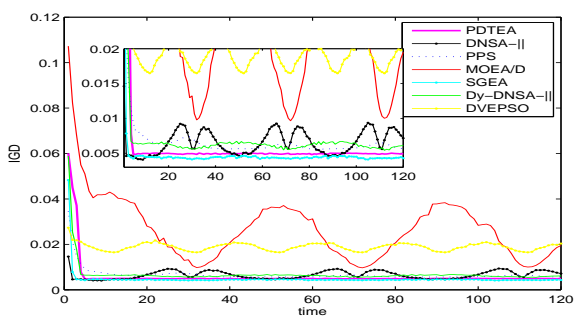

(b) FDA2

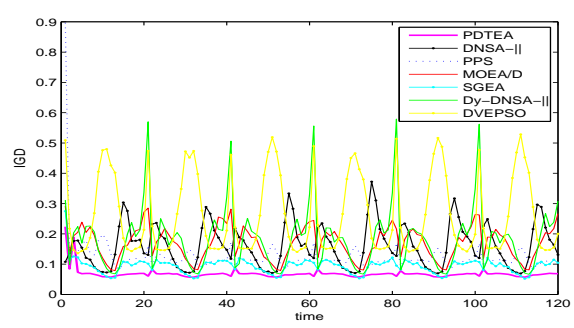

(d) FDA4

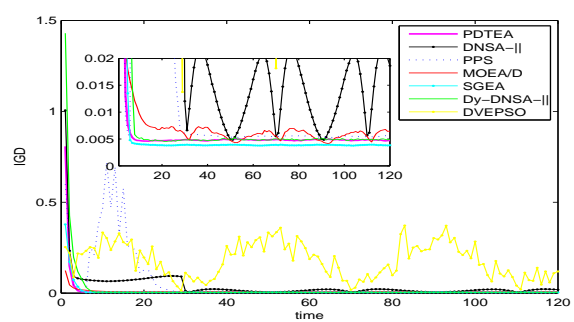

(f) dMOP1

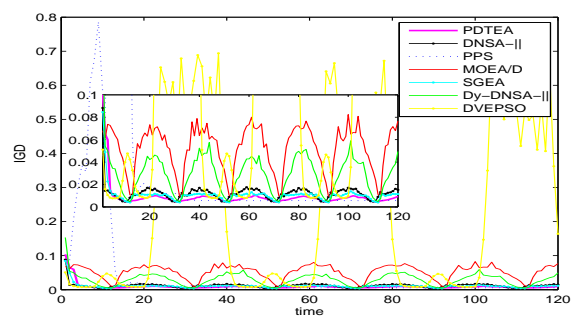

(h) dMOP3

Figure 5: Evolution curves of average IGD values for eight problems with $n_{T}$ $=10$ and $\tau_{T}=30$. 


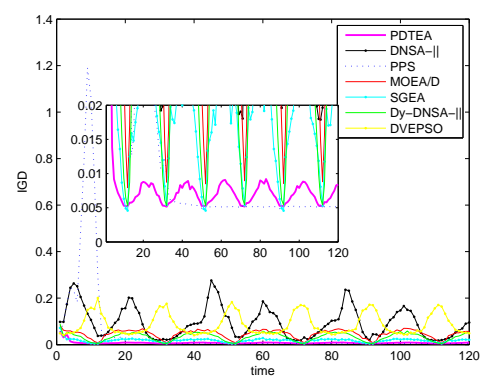

(a) JY1

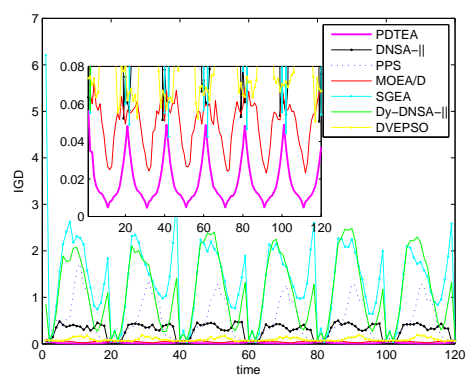

(d) JY4

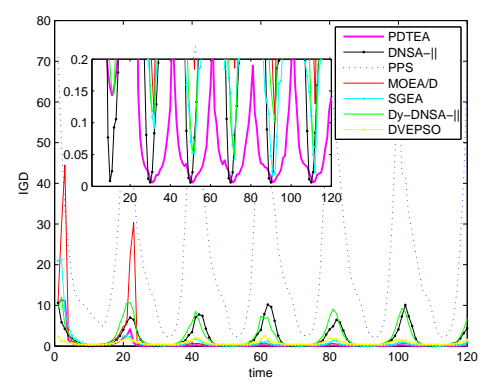

(g) JY7

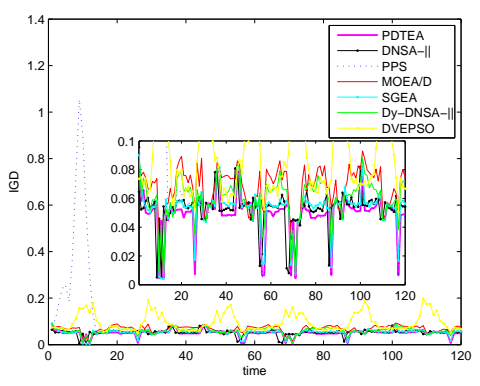

(b) JY2

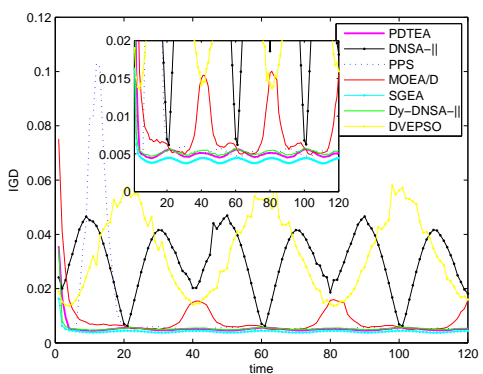

(e) JY5

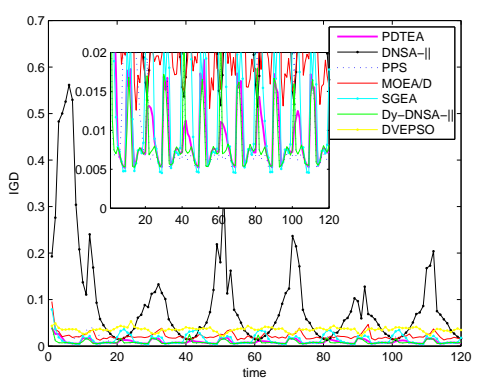

(h) JY8

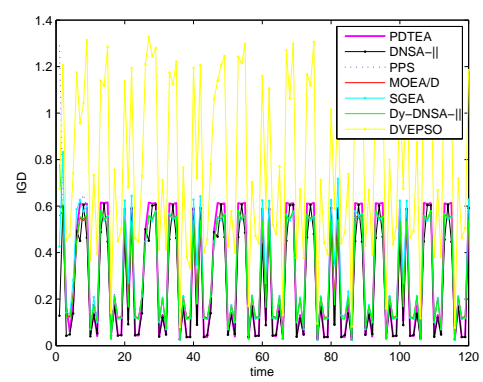

(c) JY3

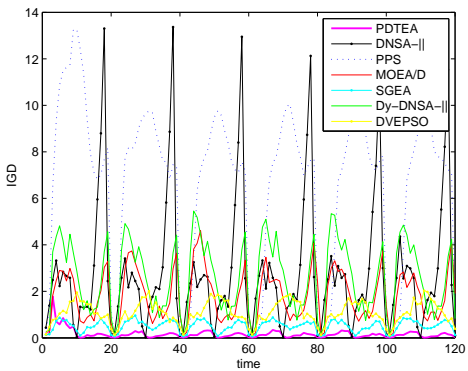

(f) JY6

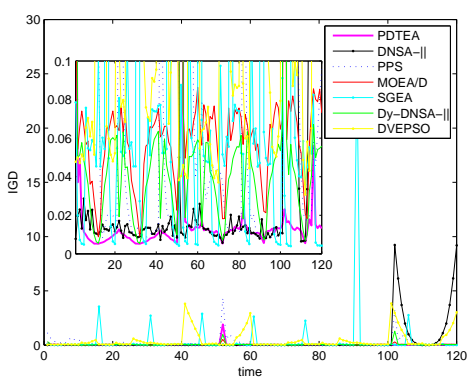

(i) JY9

Figure 6: Evolution curves of average IGD values for nine problems with $n_{T}$ $=10$ and $\tau_{T}=30$.

\subsection{Influence of severity of change}

Severity of change $\left(n_{t}\right)$ is a critical parameter in the dynamic environment, and it can affect an algorithms' performance. In the experiments, $\tau_{t}$ was fixed to 

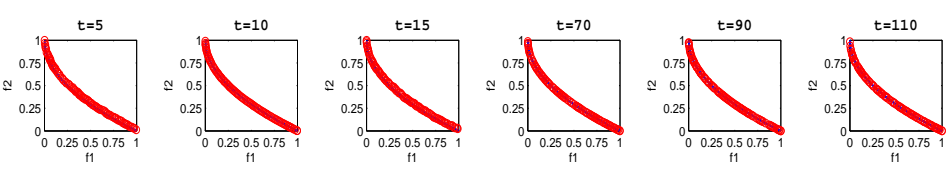

(a) DNSGA-II
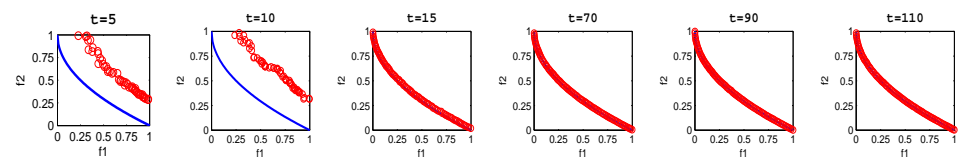

(b) PPS
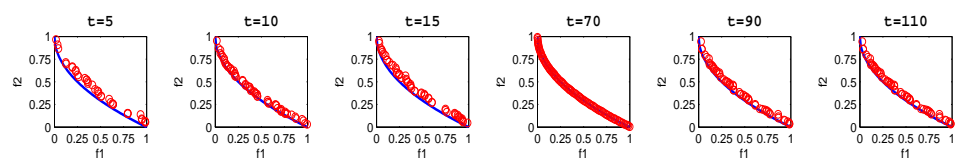

(c) MOEA/D
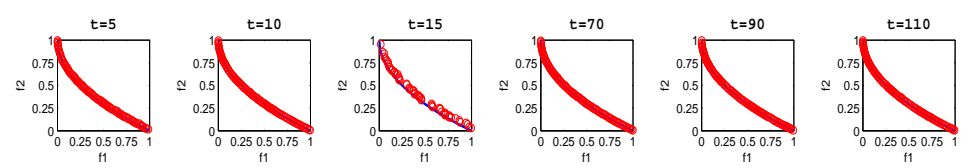

(d) SGEA
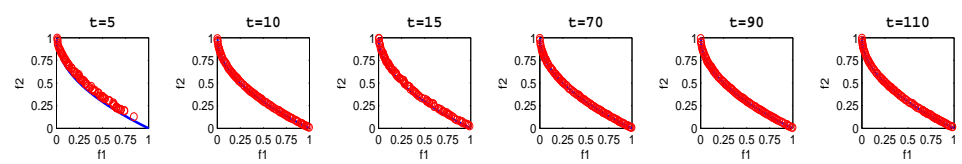

(e) Dy-NSGA- $I I$
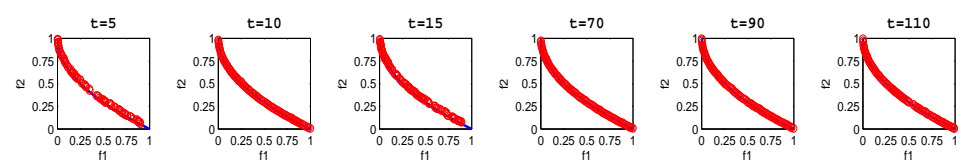

(f) DVEPSO
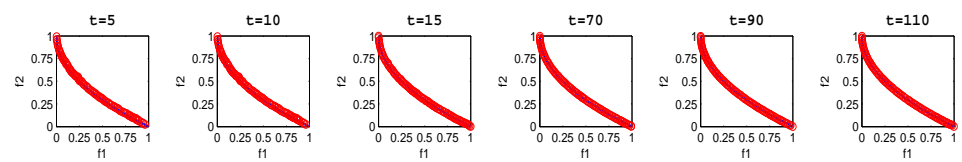

(g) PDTEA

Figure 7: Solution sets obtained by seven algorithms at six different time steps on FDA1. 

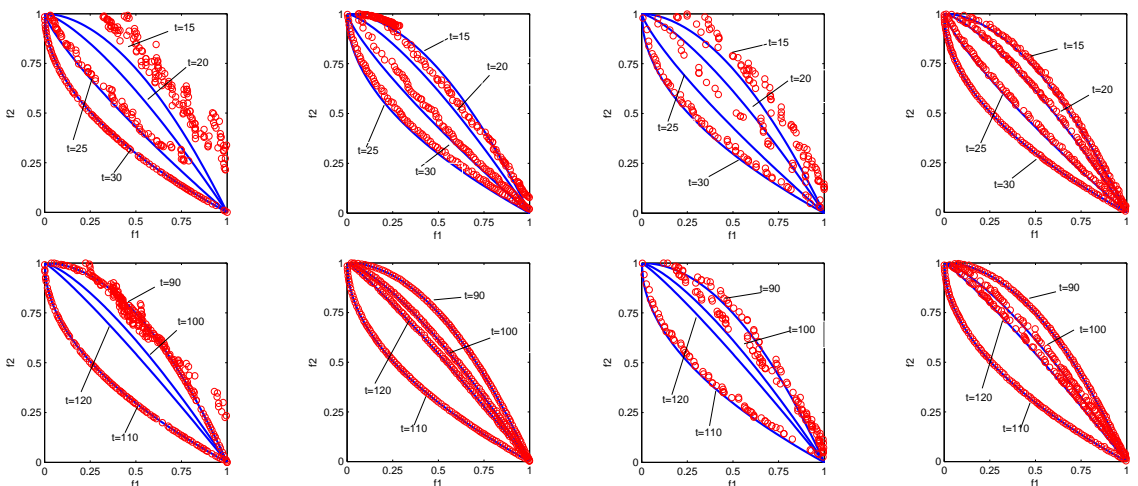

(b) PPS

(c) MOEAD

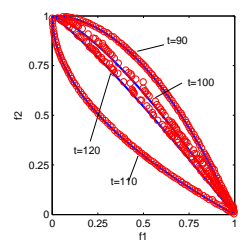

(a) DNSGA-II
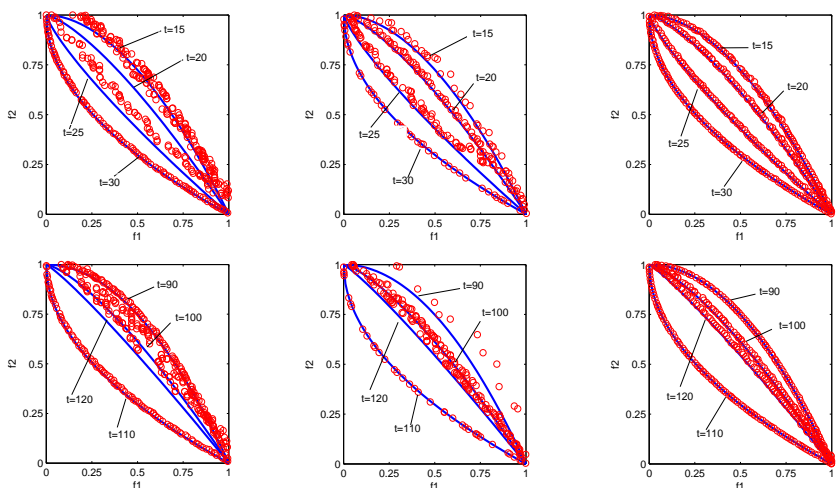

(e) D-NSGA-II

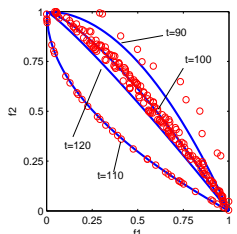

(f) DVEPSO

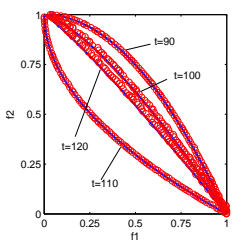

(g) PDTEA

Figure 8: Solution sets obtained by seven algorithms at eight different time steps on dMOP2.

25 , and $n_{t}$ was set to 5,10 and 20, for severe, moderate, and slight environmental changes, respectively. The statistical results of HVD for each algorithm are shown in Table 5 and we can observe sensitivity to the severity of change.

It is clear that severity of change can greatly affect the algorithms' performance, and algorithms have better performance when increasing the value of $n_{t}$. In most cases, PDTEA had better performance. However, PDTEA was surpassed by SGEA in three problems, which were FDA2, dMOP1 and JY5. The reason, as previously explained, is that SGEA has great superiority when solving problems with fixed POS due to the use of half of past solutions. PDTEA is 

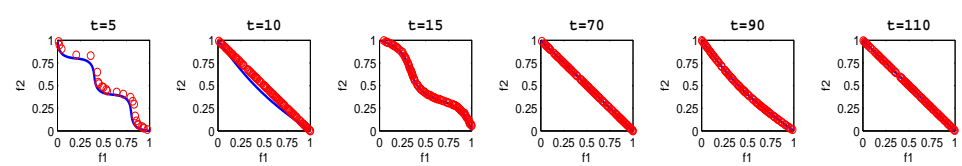

(a) DNSGA $I I$
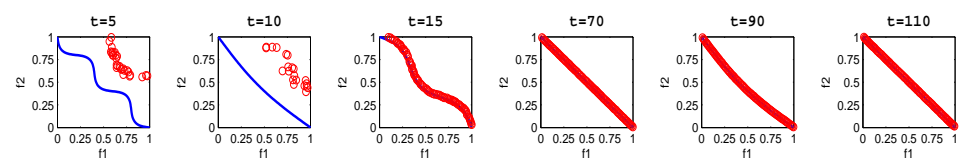

(b) PPS
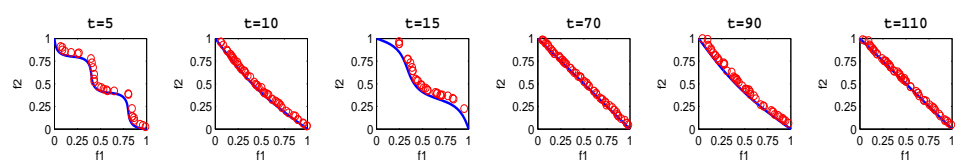

(c) MOEAD
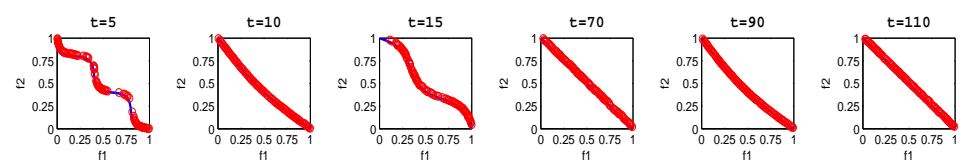

(d) SGEA
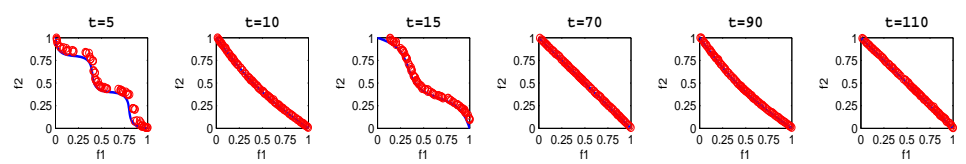

(e) D-NSGA-II
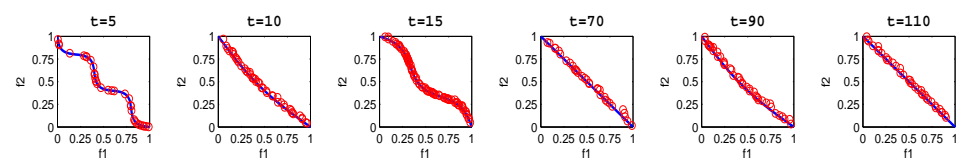

(f) DVEPSO
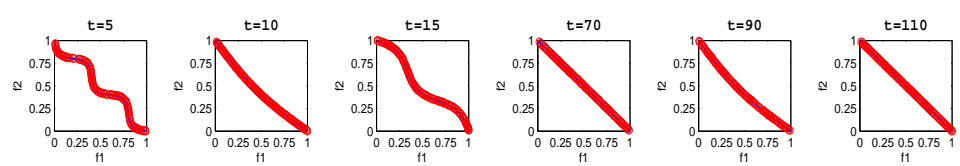

(g) PDTEA

Figure 9: Solution sets obtained by seven algorithms at six different time steps on JY2. 


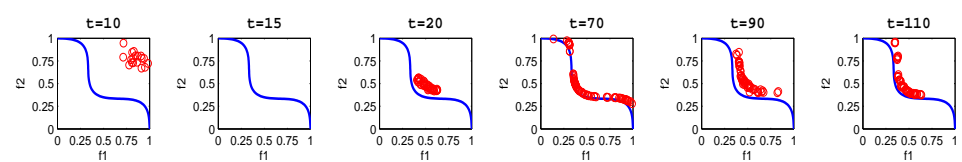

(a) DNSGAII
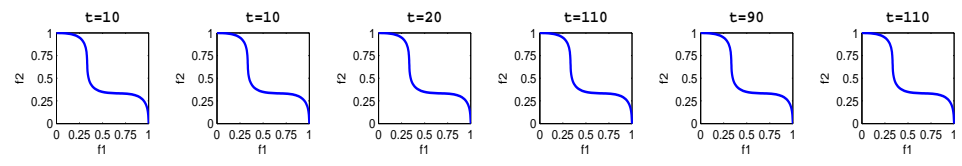

(b) PPS
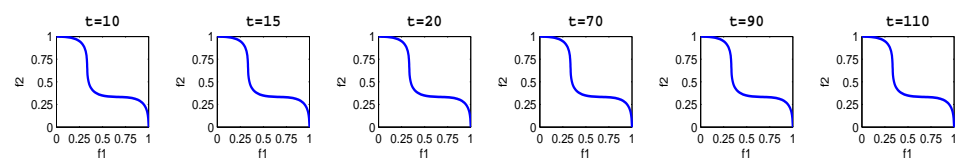

(c) MOEAD
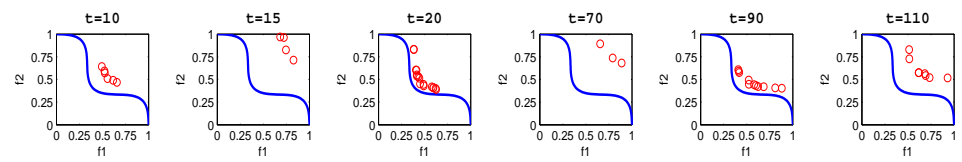

(d) SGEA
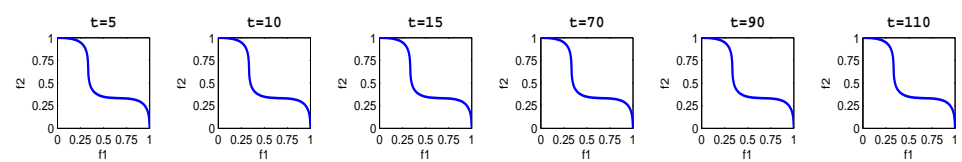

(e) D-NSGA-II
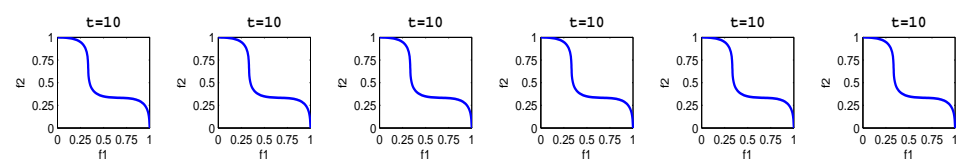

(f) DVEPSO
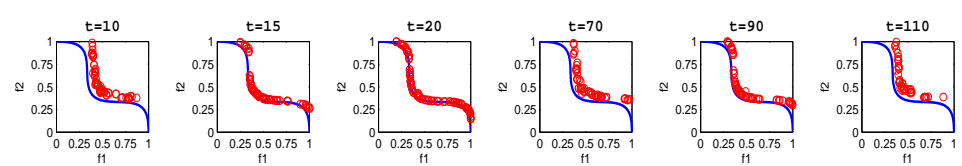

(g) PDTEA

Figure 10: Solution sets obtained by seven algorithms at six different time steps on JY6. 
Table 5: Mean and SD of HVD indicator obtained by seven algorithms.

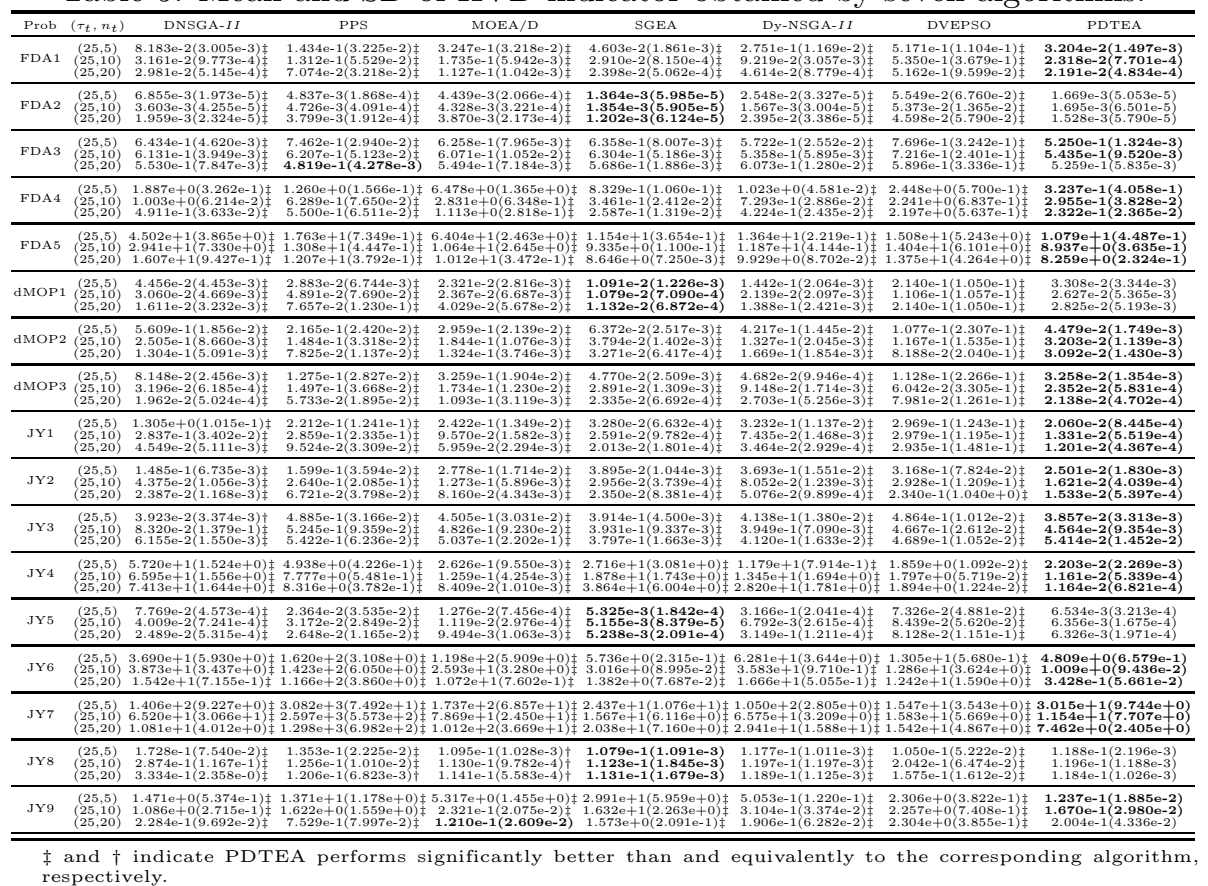

less sensitive as the severity of change increases. The reason might be because the exploration and exploitation strategies are used to find the new POS and it can quickly react to the change of environment. But for JY9, as $n_{t}$ increases, the performances of PDTEA decreases. The likely explanation is that JY9 is a novel problem that cyclically switches from type $I$ to $I I$, then to type $I I I$ 2] and the frequency of type changes also influences the performance of the algorithms. It therefore poses a new challenge that the algorithm needs a mix of solutions with good diversity and convergence. PDTEA possibly needs a new 725 method to deal with the problem.

\subsection{Study of different components of PDTEA}

PDTEA consists of three critical components including a dynamic handling mechanism to respond to the environmental changes, mating selection and environmental selection. In order to study the role of each component in dynamic optimization, the original PDTEA was transformed to three versions. The first 
Table 6: Mean and SD of GD, IGD and HVD indicator obtained by four algorithms

\begin{tabular}{|c|c|c|c|c|c|}
\hline Problem & Indicator & PDTEA-S1 & PDTEA-S2 & PDTEA-S3 & PDTEA \\
\hline FDA1 & $\begin{array}{l}\text { GD } \\
\text { IGD } \\
\text { HVD }\end{array}$ & $\begin{array}{l}4.846 \mathrm{e}-2(8.728 \mathrm{e}-4) \ddagger \\
5.228 \mathrm{e}-2(2.548 \mathrm{e}-3) \\
1.129 \mathrm{e}-1(2.184 \mathrm{e}-3)\end{array}$ & $\begin{array}{l}9.373 \mathrm{e}-3(4.516 \mathrm{e}-4) \ddagger \\
1.039 \mathrm{e}-2(1.125 \mathrm{e}-3) \\
2.381 \mathrm{e}-2(5.390 \mathrm{e}-4)\end{array}$ & $\begin{array}{l}2.043 \mathrm{e}-2(3.262 \mathrm{e}-3) \ddagger \\
1.248 \mathrm{e}-2(1.605 \mathrm{e}-3) \\
2.423 \mathrm{e}-2(8.443 \mathrm{e}-4)\end{array}$ & $\begin{array}{l}8.455 \mathrm{e}-3(3.708 \mathrm{e}-4) \\
1.018 \mathrm{e}-2(3.993 \mathrm{e}-4) \\
2.318 \mathrm{e}-2(7.701 \mathrm{e}-4)\end{array}$ \\
\hline FDA4 & $\begin{array}{l}\text { GD } \\
\text { IGD } \\
\text { HVD }\end{array}$ & $\begin{array}{c}3.580 \mathrm{e}-1(1.031 \mathrm{e}-2) \ddagger \\
2.689 \mathrm{e}-1(6.976 \mathrm{e}-3) \ddagger \\
3.759 \mathrm{e}+0(3.403 \mathrm{e}-1) \ddagger\end{array}$ & $\begin{array}{l}4.731 \mathrm{e}-2(1.902 \mathrm{e}-3) \ddagger \\
7.617 \mathrm{e}-2(1.111 \mathrm{e}-3) \\
3.171 \mathrm{e}-1(2.032 \mathrm{e}-2)\end{array}$ & $\begin{array}{c}2.095 \mathrm{e}-1(2.027 \mathrm{e}-2) \ddagger \\
8.623 \mathrm{e}-2(1.666 \mathrm{e}-3) \ddagger \\
9.531 \mathrm{e}+0(2.610 \mathrm{e}+0) \ddagger\end{array}$ & $\begin{array}{l}4.196 \mathrm{e}-2(4.196 \mathrm{e}-2) \\
7.122 \mathrm{e}-2(6.816 \mathrm{e}-4) \\
2.748 \mathrm{e}-1(3.788 \mathrm{e}-2)\end{array}$ \\
\hline dMOP2 & $\begin{array}{l}\text { GD } \\
\text { IGD } \\
\text { HVD }\end{array}$ & $\begin{array}{l}7.227 \mathrm{e}-2(1.845 \mathrm{e}-3) \ddagger \\
6.465 \mathrm{e}-2(1.687 \mathrm{e}-3) \\
1.663 \mathrm{e}-1(4.807 \mathrm{e}-3)\end{array}$ & $\begin{array}{l}1.378 \mathrm{e}-2(5.748 \mathrm{e}-4) \\
3.110 \mathrm{e}-2(3.710 \mathrm{e}-4) \\
3.803 \mathrm{e}-2(1.146 \mathrm{e}-3)\end{array}$ & $\begin{array}{c}1.977 \mathrm{e}-2(2.413 \mathrm{e}-3) \ddagger \\
1.284 \mathrm{e}-2(9.086 \mathrm{e}-4) \dagger \\
\mathbf{3 . 1 3 7 e - 2}(\mathbf{1 . 4 2 4 e - 3})\end{array}$ & $\begin{array}{c}1.133 \mathrm{e}-2(5.974 \mathrm{e}-4) \\
1.264 \mathrm{e}-2(8.063 \mathrm{e}-4) \\
3.203 \mathrm{e}-2(1.139 \mathrm{e}-3)\end{array}$ \\
\hline JY2 & $\begin{array}{l}\text { GD } \\
\text { IGD } \\
\text { HVD }\end{array}$ & $\begin{array}{l}6.520 \mathrm{e}-2(8.731 \mathrm{e}-4) \ddagger \\
6.367 \mathrm{e}-2(6.016 \mathrm{e}-4) \\
8.634 \mathrm{e}-2(2.049 \mathrm{e}-3) \ddagger\end{array}$ & $\begin{array}{l}4.921 \mathrm{e}-2(2.469 \mathrm{e}-4) \dagger \\
5.116 \mathrm{e}-2(2.632 \mathrm{e}-4) \\
1.712 \mathrm{e}-2(6.111 \mathrm{e}-4)\end{array}$ & $\begin{array}{l}6.910 \mathrm{e}-2(2.289 \mathrm{e}-3) \ddagger \\
5.034 \mathrm{e}-2(8.467 \mathrm{e}-5) \dagger \\
1.833 \mathrm{e}-2(2.474 \mathrm{e}-4) \ddagger\end{array}$ & $\begin{array}{l}4.922 \mathrm{e}-2(4.922 \mathrm{e}-2) \\
5.024 \mathrm{e}-2(1.327 \mathrm{e}-4) \\
1.621 \mathrm{e}-2(4.039 \mathrm{e}-4)\end{array}$ \\
\hline JY3 & $\begin{array}{l}\text { GD } \\
\text { IGD } \\
\text { HVD }\end{array}$ & $\begin{array}{l}2.532 \mathrm{e}-1(5.274 \mathrm{e}-3) \ddagger \\
3.193 \mathrm{e}-1(3.121 \mathrm{e}-3) \\
4.007 \mathrm{e}-1(7.498 \mathrm{e}-3)\end{array}$ & $\begin{array}{c}\mathbf{7 . 5 6 9 e - 2}(3.502 \mathrm{e}-3) \\
3.194 \mathrm{e}-1(2.857 \mathrm{e}-3) \dagger \\
4.804 \mathrm{e}-2(8.135 \mathrm{e}-3) \ddagger\end{array}$ & $\begin{array}{l}1.019 \mathrm{e}-1(3.161 \mathrm{e}-2) \ddagger \\
3.099 \mathrm{e}-1(1.846 \mathrm{e}-3) \dagger \\
5.134 \mathrm{e}-2(8.335 \mathrm{e}-3) \ddagger\end{array}$ & $\begin{array}{c}7.582 \mathrm{e}-2(3.673 \mathrm{e}-3) \\
\mathbf{3 . 0 8 9 e - 1}(\mathbf{2 . 9 8 8 e - 3}) \\
\mathbf{4 . 5 6 4 e - 2}(\mathbf{9 . 3 5 4 e - 3})\end{array}$ \\
\hline JY5 & $\begin{array}{l}\text { GD } \\
\text { IGD } \\
\text { HVD }\end{array}$ & $\begin{array}{l}1.483 \mathrm{e}-3(7.523 \mathrm{e}-5) \dagger \\
5.516 \mathrm{e}-3(1.491 \mathrm{e}-4) \dagger \\
6.414 \mathrm{e}-3(1.149 \mathrm{e}-4) \ddagger\end{array}$ & $\begin{array}{l}1.532 \mathrm{e}-3(7.385 \mathrm{e}-5) \dagger \\
5.655 \mathrm{e}-3(5.158 \mathrm{e}-5) \\
6.458 \mathrm{e}-3(1.217 \mathrm{e}-4)\end{array}$ & $\begin{array}{l}4.532 \mathrm{e}-3(1.630 \mathrm{e}-3) \ddagger \\
5.550 \mathrm{e}-3(6.510 \mathrm{e}-5) \dagger \\
6.800 \mathrm{e}-3(2.193 \mathrm{e}-4)\end{array}$ & $\begin{array}{l}1.481 \mathrm{e}-3(1.031 \mathrm{e}-4) \\
5.454 \mathrm{e}-3(1.298 \mathrm{e}-4) \\
6.356 \mathrm{e}-3(1.675 \mathrm{e}-4)\end{array}$ \\
\hline JY8 & $\begin{array}{l}\text { GD } \\
\text { IGD } \\
\text { HVD }\end{array}$ & $\begin{array}{l}6.199 \mathrm{e}-3(3.711 \mathrm{e}-4) \\
1.483 \mathrm{e}-2(1.940 \mathrm{e}-3) \\
1.165 \mathrm{e}-1(1.657 \mathrm{e}-3)\end{array}$ & $\begin{array}{r}6.333 \mathrm{e}-3(6.022 \mathrm{e}-4) \\
\mathbf{1 . 0 5 4 e - 2}(\mathbf{7 . 2 4 4})-4 \\
\mathbf{1 . 1 1 6 e - 1}(\mathbf{2 . 0 7 0 e - 3})\end{array}$ & $\begin{array}{l}1.328 \mathrm{e}-2(1.699 \mathrm{e}-3) \ddagger \\
1.073 \mathrm{e}-2(6.697 \mathrm{e}-4) \dagger \\
1.183 \mathrm{e}-1(9.848 \mathrm{e}-4) \dagger\end{array}$ & $\begin{array}{c}\mathbf{6 . 0 7 5 e - 3 ( 4 . 1 4 8 e - 4 )} \\
1.068 \mathrm{e}-2(7.110 \mathrm{e}-4) \\
1.196 \mathrm{e}-1(1.188 \mathrm{e}-3)\end{array}$ \\
\hline
\end{tabular}

$\ddagger$ and $\dagger$ indicate PDTEA performs significantly better than and equivalently to the corresponding algorithm, respectively.

version (PDTEA-s1) does not use the dynamic handling mechanism to respond to environmental changes, and it re-evaluates the current population whenever the environmental change occurs. The second version (PDTEA-s2) uses tournament selection to replace mating selection. PDTEA-s3 utilizes nondominated sort and crowding distance and then selects $\mathrm{N}$ individuals as environmental selection. These three variants were compared with the original PDTEA with the setting of $\left(\tau, n_{t}\right)=(25,10)$. The statistical results are shown in Table 6 including the average and standard deviation values of three metrics. The Wilcoxon rank-sum [59] is set to the 0.05 significance level.

As can be seen from Table 6. PDTEA significantly surpassed other versions in most problems including FDA1, FDA4 and JY2, indicating that each component is essential and indispensable for enhancing the performance of PDTEA. For dMOP2, PDTEA surpassed others in terms of GD and IGD values, while PDTEA-S3 outperformed PDTEA with respect to the HVD indicator. One 745 possible explanation is that PDTEA-S3 mainly adopts the crowding distance other than the truncation operator to maintain diversity. Specifically, the former is beneficial for the algorithms to preserve the boundary points, while the 
latter does not have this benefit in the critical layer during the latter period of optimization. Moreover, the preservation of boundary points can contribute to the decrease of the HVD value. As for JY3, PDTEA significantly exceeded the other versions in terms of IGD and HVD. However, the GD value of PDTEA is not as good as that of PDTEA-S2. Combining these two discoveries, we can conclude that PDTEA can maintain adequate diversity when conducting the mating selection, which is good for the algorithms' diversity and convergence, presented on the values of HVD and IGD values. Nevertheless, overuse of diversity introduction may lead to low convergence, which can be found on the GD values of PDTEA-S2. For JY5 whose POS remains unchanged, PDTEA showed better performance than PDTEA-S3 in terms of all selected indicators, with equal performance to PDTEA-S1 and PDTEA-S2. The conclusion can be drawn that the dynamic handling mechanism and mating selection do not have such big benefits when solving problems with fixed POS. The reason might be that the diversity introduced by these two strategies may misguide the evolution of the population. When it comes to JY8, PDTEA was better than the others in terms of GD, indicating that the three mechanisms are beneficial for the convergence of PDTEA. However, PDTEA performed worse than PDTEA-S2 and equal to PDTEA-S3 in terms of IGD and HVD values, which suggests that the mating selection and truncation operation of PDTEA do not have much help to enhance the diversity of population when solving problems whose geometry and number of mixed segments of POF vary over time.

It can be concluded that PDTEA, consisting of three key components, is superior to other modified versions. The finding clearly demonstrates the significant and indispensable role of each component in coping with a dynamic environment. The role that different parts play in PDTEA will be further explained. In order to enhance the diversity of the population during optimization, different well-diversified solutions from different regions of the associated reference points are chosen as the mating parents in the mating selection. It therefore maintains the overall diversity of the population. Additionally, as an excellent selection strategy which has been demonstrated, the Pareto-based 
dominance selection strategy is applied to choose first-class offspring solutions, so as to speed up the overall convergence of the population during the evolution. Besides that, the modified truncation operation is used to estimate the density of the population from an overall perspective. Eventually, in order to quickly and accurately trace the changing environment, the exploration and exploitation strategies in the dynamic handling mechanism are adopted. The former one is to explore the possible area in which the new population may locate, thereby responding to the environmental changes in a quick manner, which can also maintain the diversity of population to some degree. The latter one is used to generate some some well-converged and well-diversified solutions around the situated POS of the next environmental changes. The algorithm can therefore exploit the promising area adequately and completely. The dynamic handling strategy is able to enhance the population diversity in the responding stage. Overall, diversity and convergence can be simultaneously achieved both during evolution and in the environmental changes' response phrases.

\subsection{Study of different dimensions of the decision space}

In order to study the influence of the decision space size on algorithms' performance, some experiments were conducted on FDA1, FDA4, dMOP2, JY1, JY5 and JY9. The relevant parameter settings are described as $\tau_{t}=25, n_{t}=10$ and $n=10,20$ and 30 , respectively. The statistical results of HVD for each algorithm are presented in Table 7.

In Table 7, PDTEA is shown to have obtained a better performance on a majority of problems than other algorithms except JY5. Moreover, it can be clearly seen from Table 7 that the size of the decision space can significantly affect the algorithms' performance. For most problems, the algorithms' performance is sensitive to the size of the decision space and the effect is aggravated when the size of the decision space becomes bigger and bigger. Overall, the size of the decision space plays an important role in affecting the algorithms' performances. When size is decreased, the algorithm can obtain good performance. 
Table 7: Mean and SD of HVD indicator obtained by seven algorithms.

\begin{tabular}{|c|c|c|c|c|c|c|c|c|}
\hline Prob & $\mathrm{n}$ & DNSGA- $I I$ & PPS & MOEA/D & SGEA & Dy-NSGA- $I I$ & DVEPSO & PDTEA \\
\hline & & $\begin{array}{l}.381 \mathrm{e}-2(1.010 \mathrm{e}-4) \neq \\
3.161 \mathrm{e}-2(9.7973 \mathrm{e}-4) \neq \\
3.255 \mathrm{e}-2(1.124 \mathrm{e}-3) \neq\end{array}$ & $\begin{array}{l}3.242 \mathrm{e}-2(1.702 \mathrm{e}-2) \neq \\
1.312 \mathrm{e}-1(5.529 \mathrm{e}-2) \neq \\
4.734 \mathrm{e}-1(1.038 \mathrm{e}-1) \neq\end{array}$ & $\begin{array}{l}9.614 \mathrm{e}-2(8.779 \mathrm{e}-4) \dagger \\
1.735 \mathrm{e}-1(5.952 \mathrm{e}-3) \ddagger \\
9.221 \mathrm{e}-2(2.098 \mathrm{e}-2) \neq\end{array}$ & $\begin{array}{l}1.231 \mathrm{e}-2(1.035 \mathrm{e}-4) \ddagger \\
2.910 \mathrm{e}-2(8.150 \mathrm{e}-4) \neq \\
5.826 \mathrm{e}-2(6.508 \mathrm{e}-4) \neq\end{array}$ & $\begin{array}{l}4.614 \mathrm{e}-2(8.779 \mathrm{e}-4) \neq \\
9.219 \mathrm{e}-1(3.057 \mathrm{e}-3) \neq \\
1.382 \mathrm{e}+0(1.443 \mathrm{e}-1) \neq\end{array}$ & $\begin{array}{l}1.183 \mathrm{e}-1(2.870 \mathrm{e}-2) \neq \\
5.350 \mathrm{e}-1(3.679 \mathrm{e}-1) \neq \\
\$ 8.080 \mathrm{e}-1(5.695 \mathrm{e}-1) \neq\end{array}$ & $\begin{array}{l}1.020 \mathrm{e}-2(4.625 \mathrm{e}-4) \\
2.318 \mathrm{e}-2(7.701 \mathrm{e}-4) \\
3.591 \mathrm{e}-2(8.950 \mathrm{e}-4)\end{array}$ \\
\hline & & $\begin{array}{l}1.883 \mathrm{e}-1(4.208 \mathrm{e}-2) \ddagger \\
.003 \mathrm{e}+0(6,214 \mathrm{e}-2) \ddagger \\
.882 \mathrm{e}+0(8.413 \mathrm{e}-2) \ddagger\end{array}$ & $\begin{array}{l}3.055 \mathrm{e}-1(1.415 \mathrm{e}-2) \ddagger \\
6.289 \mathrm{e}-1(7.650 \mathrm{e}-2) \dagger \\
1.453 \mathrm{e}+0(1.482 \mathrm{e}-1) \ddagger\end{array}$ & $\begin{array}{l}4.224 \mathrm{e}-1(2.435 \mathrm{e}-2) \ddagger \\
2.831 \mathrm{e}+0(6.348 \mathrm{e}-1) \neq \\
4.228 \mathrm{e}+0(8.993 \mathrm{e}-2) \ddagger\end{array}$ & $\begin{array}{l}2.135 \mathrm{e}-1(7.402 \mathrm{e}-3) \neq \\
3.461 \mathrm{e}-1(2.412 \mathrm{e}-2) \neq \\
8.860 \mathrm{e}-1(8.931 \mathrm{e}-2) \neq\end{array}$ & $\begin{array}{l}4.224 \mathrm{e}-1(2.435 \mathrm{e}-2) \neq \\
7.293 \mathrm{e}-1(2.886 \mathrm{e}-2) \\
2.244 \mathrm{e}+0(1.994 \mathrm{e}-1) \neq\end{array}$ & 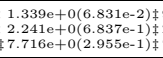 & $\begin{array}{l}\$ 9.154 \mathrm{e}-2(3.805 \mathrm{e}-2) \\
\$ 2.748 \mathrm{e}-1(3.788 \mathrm{e}-2) \\
\$ 7.684 \mathrm{e}-1(8.104 \mathrm{e}-2)\end{array}$ \\
\hline & 105 & $\begin{array}{l}5.751 \mathrm{e}-2(2.333 \mathrm{e}-3) \neq \\
2.505 \mathrm{e}-1(8.660 \mathrm{e}-3) \neq \\
5.423 \mathrm{e}-1(1.815 \mathrm{e}-2) \neq\end{array}$ & $\begin{array}{l}3.333 \mathrm{e}-2(1.165 \mathrm{e}-2) \neq \\
1.448 \mathrm{e}-1(3.318 \mathrm{e}-2) \neq \\
3.563 \mathrm{e}-1(4.873 \mathrm{e}-2) \neq\end{array}$ & $\begin{array}{l}1.669 \mathrm{e}-1(1.854 \mathrm{e}-3) \neq \\
1.84 \mathrm{e}-1(1.076 \mathrm{e}-3) \neq \\
2.185 \mathrm{e}-1(3.719 \mathrm{e}-3) \neq\end{array}$ & $\begin{array}{l}1.601 \mathrm{e}-2(9.204 \mathrm{e}-4) \ddagger \\
3.794 \mathrm{e}-2(1.402 \mathrm{e}-3) \neq \\
7.870 \mathrm{e}-2(2.237 \mathrm{e}-3) \ddagger\end{array}$ & $\begin{array}{l}1.669 \mathrm{e}-1(1.854 \mathrm{e}-3) \neq \\
1.327 \mathrm{e}-1(2.045 \mathrm{e}-3) \neq \\
1.573 \mathrm{e}+0(1.757 \mathrm{e}-1) \neq\end{array}$ & $\begin{array}{l}4.672 \mathrm{e}-2(5.196 \mathrm{e}-2) \neq \\
1.167 \mathrm{e}-1(1.535 \mathrm{e}-1) \neq \\
4.916 \mathrm{e}-1(1.888 \mathrm{e}-1) \neq\end{array}$ & $\begin{array}{l}1.204 \mathrm{e}-2(3.930 \mathrm{e}-4) \\
3.203 \mathrm{e}-2(1.139 \mathrm{e}-3) \\
4.618 \mathrm{e}-2(3.519 \mathrm{e}-3)\end{array}$ \\
\hline JY1 & & $\begin{array}{l}3.235 \mathrm{e}-2(3.464 \mathrm{e}-3) \dagger \\
2.837 \mathrm{e}-1(3.402 \mathrm{e}-2) \ddagger \\
.031 \mathrm{e}+0(2.424 \mathrm{e}-1) \dagger\end{array}$ & $\begin{array}{l}1.575 \mathrm{e}-2(1.610 \mathrm{e}-2) \dagger \\
2.889 \mathrm{e}-1(2.335 \mathrm{e}-1) \ddagger \\
1.224 \mathrm{e}+0(8.92 \mathrm{e}-1) \dagger\end{array}$ & $\begin{array}{l}3.464 \mathrm{e}-2(2.929 \mathrm{e}-4) \dagger \\
9.570 \mathrm{e}-2(1.582 \mathrm{e}-3) \\
6.957 \mathrm{e}-1(1.359 \mathrm{e}-3) \dagger\end{array}$ & $\begin{array}{l}8.771 \mathrm{e}-3(5.104 \mathrm{e}-4) \dagger \\
2.551 \mathrm{e}-2(9.782 \mathrm{e}-4) \neq \\
5.907 \mathrm{e}-2(1.687 \mathrm{e}-3) \dagger\end{array}$ & $\begin{array}{l}3.464 \mathrm{e}-2(2.929 \mathrm{e}-4) \dagger \\
7.435 \mathrm{e}-2(1.468 \mathrm{e}-3) \\
9.720 \mathrm{e}-1(1.986 \mathrm{e}-1) \dagger\end{array}$ & $\begin{array}{l}8.151 \mathrm{e}-2(3.620 \mathrm{e}-3) \neq \\
2.979 \mathrm{e}-1(1.1195 \mathrm{e}-1) \neq \\
6.871 \mathrm{e}-1(3.485 \mathrm{e}-1) \neq\end{array}$ & $\begin{array}{l}4.747 \mathrm{e}-3(3.274 \mathrm{e}-5) \\
1.331 \mathrm{e}-2(5.519 \mathrm{e}-4) \\
1.855 \mathrm{e}-2(4.562 \mathrm{e}-4)\end{array}$ \\
\hline JY5 & & $\begin{array}{l}3.883 \mathrm{e}-2(2.413 \mathrm{e}-4) \neq \\
.009 \mathrm{e}-2(7.241 \mathrm{e}-4) \neq \\
1.178 \mathrm{e}-2(1.347 \mathrm{e}-3) \neq\end{array}$ & $\begin{array}{l}8.555 \mathrm{e}-3(7.394 \mathrm{e}-5) \neq \\
3.172 \mathrm{e}-2(2.8499 \mathrm{e}-2) \neq \\
8.037 \mathrm{e}-2(8.027 \mathrm{e}-2) \neq\end{array}$ & $\begin{array}{l}9.149 \mathrm{e}-3(1.211 \mathrm{e}-4) \neq \\
1.119 \mathrm{e}-2(2.296 \mathrm{e}-4) \\
2.005 \mathrm{e}-2(1.782 \mathrm{e}-4) \neq \\
2.45\end{array}$ & $\begin{array}{l}4.707 \mathrm{e}-3(2.954 \mathrm{e}-5) \neq \\
5.155 \mathrm{e}-3(8.3799-5) \\
6.690 \mathrm{e}-3(3.148 \mathrm{e}-4)\end{array}$ & 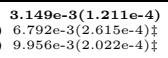 & $\begin{array}{l}4.194 \mathrm{e}-2(4.568 \mathrm{e}-2) \neq \\
8.439 \mathrm{e}-2(5.620 \mathrm{e}-2) \\
4.387 \mathrm{e}-1(7.671 \mathrm{e}-2) \neq\end{array}$ & $\begin{array}{l}4.334 \mathrm{e}-3(4.594 \mathrm{e}-5) \\
6.356 \mathrm{e}-3(1.675 \mathrm{e}-4) \\
7.837 \mathrm{e}-3(2.047 \mathrm{e}-4)\end{array}$ \\
\hline JY9 & & & & & & & & $\begin{array}{l}88 \mathrm{e}-4) \\
80-2) \\
12 \mathrm{e}-2)\end{array}$ \\
\hline
\end{tabular}
$\ddagger$ and $\uparrow$ indical
respectively

\subsection{Statistical Analysis}

In order to take into account the tracking ability of the DMOEAs, the for-

mal wins and losses approach by Helbig et al. [61] were used to compare the performance of the algorithms. All statistical tests were performed at the $95 \%$ confidence level. The relevant parameter settings are described as $\tau_{t}=25$ and $n_{t}=10$. Experimental results of seven algorithms on the IGD metric are presented in Table 8 .

It can be observed in the Table 8 that PDTEA obtained the best rank on FDA1, FDA4 and dMOP2. Therefore, the results indicate PDTEA can obtain good performance when tracking the moving POF. However, PDTEA ranks second and SGEA ranks first on JY5. SGEA obtained better IGD average values than PDTEA on most of the time steps. The reason is that SGEA can reuse half of the old solutions to adapt to a new environment.

\subsection{Study of influence on population diversity}

Introducing diversity for environmental changes is considerably important. In order to empirically study influence on population diversity, we can introduce $\eta \%$ mutated individuals [9] of current population into the new population, and use (100- $\eta) \%$ individuals by the response mechanism proposed in Algorithm3 Thus, the new population is composed of $\eta \%(0 \leq \eta \leq 100)$ mutated individuals and (100- $\eta) \%$ promising individuals generated in Algorithm 3. We call this version of PDTEA as PDTEA-v1. We select FDA1 and dMOP2 with the setting 
Table 8: Wins and Losses on FDA1, FDA4, dMOP2 and JY5 using IGD.

\begin{tabular}{ccccccccc}
\hline Prob & result & DNSGA- $I I$ & PPS & MOEA/D & SGEA & Dy-NSGA- $I I$ & DVEPSO & PDTEA \\
\hline \multirow{4}{*}{ FDA1 } & Wins & 67.56 & 73.53 & 6.47 & 70.69 & 31.09 & 49.53 & 94.88 \\
& Losses & 44.94 & 38.97 & 106.03 & 41.81 & 81.41 & 62.97 & 17.63 \\
& Diff & 22.63 & 34.56 & -99.56 & 28.88 & -50.31 & -13.44 & 77.25 \\
& Rank & 4 & 2 & 7 & 3 & 6 & 5 & 1 \\
\hline \multirow{4}{*}{ FDA4 } & Wins & 38.47 & 53.06 & 17.28 & 82.81 & 37.59 & 57.13 & 107.40 \\
& Losses & 74.03 & 59.44 & 95.22 & 29.69 & 74.91 & 55.38 & 5.09 \\
& Diff & -35.56 & -6.37 & -77.94 & 53.13 & -37.31 & 1.75 & 102.30 \\
& Rank & 5 & 4 & 7 & 2 & 6 & 3 & 1 \\
\hline \multirow{4}{*}{ dMOP2 } & Wins & 28.31 & 79.63 & 34.44 & 80.22 & 59.41 & 12.97 & 98.78 \\
& Losses & 84.19 & 32.88 & 78.06 & 32.28 & 53.09 & 99.53 & 13.72 \\
& Diff & -55.88 & 46.75 & -43.63 & 47.94 & 6.31 & -86.56 & 85.06 \\
& Rank & 6 & 3 & 5 & 2 & 4 & 7 & 1 \\
\hline \multirow{4}{*}{ JY5 } & Wins & 11.69 & 53.69 & 45.56 & 112.13 & 73.00 & 12.22 & 85.47 \\
& Losses & 100.81 & 58.81 & 66.94 & 0.38 & 39.50 & 100.28 & 27.03 \\
& Diff & -89.13 & -5.13 & -21.38 & 111.75 & 33.50 & -88.06 & 58.44 \\
\hline \hline
\end{tabular}

of $\tau_{t}=25$ and $n_{t}=10$ to study the influence on population diversity. With the variation of $\eta, \eta=0$ means PDTEA-v1 is the original PDTEA and $\eta=$ 100 indicates the new population consists entirely of mutated individuals. The statistical results of three metrics are shown in Table 9 ,

It can be seen from Table 9 that, for $\eta=0$, PDTEA-v1 gains the best performance on FDA1 and dMOP2. When $\eta$ increases, the results of PDTEA-v1 on three metrics notably decrease. For this reason, too many mutated individuals are introduced, and population diversity loss may affect the performance of algorithms.

Table 9: Mean and SD of GD, IGD and HVD indicators of PDTEA-v1 on FDA1 and dMOP2.

\begin{tabular}{|c|c|c|c|c|c|c|c|}
\hline Prob & Indicator & $\eta=0$ & $\eta=20$ & $\eta=40$ & $\eta=60$ & $\eta=80$ & $\eta=100$ \\
\hline \multirow[b]{2}{*}{ FDA1 } & $\begin{array}{l}\text { GD } \\
\text { IGD }\end{array}$ & $\begin{array}{l}8.455 \mathrm{e}-3(3.708 \mathrm{e}-4) \\
1.018 \mathrm{e}-2(3.993 \mathrm{e}-4\end{array}$ & $\begin{array}{l}8.810 \mathrm{e}-3(2.273 \mathrm{e}-4) \ddagger \\
173 \mathrm{e}-2(2.231 \mathrm{e}-3)\end{array}$ & $\begin{array}{l}8.970 \mathrm{e}-3(3.455 \mathrm{e}-4) \ddagger \\
2324 \mathrm{e}-2(1005 \mathrm{e}-3)\end{array}$ & 9. $.353 \mathrm{e}-3(1.840 \mathrm{e}-4) \ddagger$ & $9.939 \mathrm{e}-3(2.825 \mathrm{e}-4) \ddagger$ & $3.568 \mathrm{e}-2(1.099 \mathrm{e}-3) \ddagger$ \\
\hline & HVD & $\begin{array}{l}1.018 \mathrm{e}-2(3.993 \mathrm{e}-4) \\
2.318 \mathrm{e}-2(7.701 \mathrm{e}-4)\end{array}$ & $\begin{array}{l}1.713 \mathrm{e}-2(2.231 \mathrm{e}-3) \ddagger \\
2.844 \mathrm{e}-2(3.900 \mathrm{e}-4) \ddagger\end{array}$ & $\begin{array}{l}2.324 \mathrm{e}-2(1.005 \mathrm{e}-3) \ddagger \\
3.194 \mathrm{e}-2(2.335 \mathrm{e}-4) \ddagger\end{array}$ & $\begin{array}{l}2.753 \mathrm{e}-2(5.837 \mathrm{e}-4) \ddagger \\
3.544 \mathrm{e}-2(5.099 \mathrm{e}-4) \ddagger\end{array}$ & $\begin{array}{l}3.445 \mathrm{e}-2(9.804 \mathrm{e}-4) \ddagger \\
4.120 \mathrm{e}-2(5.791 \mathrm{e}-4) \ddagger\end{array}$ & $\begin{array}{l}4.547 \mathrm{e}-2(2.119 \mathrm{e}-3) \ddagger \\
9.376 \mathrm{e}-2(1.034 \mathrm{e}-3) \ddagger\end{array}$ \\
\hline \multirow{3}{*}{ dMOP2 } & GD & $1.133 \mathrm{e}-2(5.974 \mathrm{e}-4)$ & $1.495 \mathrm{e}-2(4.809 \mathrm{e}-4) \ddagger$ & $1.913 \mathrm{e}-2(5.144 \mathrm{e}-4) \ddagger$ & $2.205 \mathrm{e}-3(3.691 \mathrm{e}-4) \ddagger$ & $2.845 \mathrm{e}-2(3.434 \mathrm{e}-4) \ddagger$ & $5.235 \mathrm{e}-2(8.778 \mathrm{e}-4) \ddagger$ \\
\hline & IGD & $1.264 \mathrm{e}-2(8.063 \mathrm{e}-4)$ & $1.713 \mathrm{e}-2(2.231 \mathrm{e}-3) \ddagger$ & $2.154 \mathrm{e}-2(6.776 \mathrm{e}-4) \ddagger$ & $2.550 \mathrm{e}-2(6.462 \mathrm{e}-4) \ddagger$ & $3.257 \mathrm{e}-2(1.047 \mathrm{e}-3) \ddagger$ & $5.029 \mathrm{e}-2(1.863 \mathrm{e}-3) \ddagger$ \\
\hline & HVD & $3.203 \mathrm{e}-2(1.139 \mathrm{e}-3)$ & $4.178 \mathrm{e}-2(1.197 \mathrm{e}-3) \ddagger$ & $4.824 \mathrm{e}-2(1.430 \mathrm{e}-3) \ddagger$ & $4.946 \mathrm{e}-2(1.061 \mathrm{e}-3) \ddagger$ & $5.132 \mathrm{e}-2(6.991 \mathrm{e}-4) \ddagger$ & $1.308 \mathrm{e}-1(2.406 \mathrm{e}-3) \ddagger$ \\
\hline
\end{tabular}

\subsection{Study of influence on exploration}

In order to investigate the influence of the deviation of the Gaussian distribution on exploration, some experiments were conducted on JY1, JY2 and JY6 with the setting of $\tau_{t}=25$ and $n_{t}=10$. The deviation of Eq. 14 was set to $0, \sigma_{t}$, 
$5 \sigma_{t}$ and $10 \sigma_{t}$. In response change, we only use the exploration strategy without the exploitation strategy. The version of PDTEA is denoted as PDTEA-v2.

The experimental results about three metric values by different deviation of the Gaussian distribution are list in Table 10. It can be observed that when $\sigma=\sigma_{t}$, PDTEA-v2 gains the best results on the JY1, JY2 and JY6, implying that the exploration is quite vulnerable to the influence of deviation. When the deviation is set as $0,5 \sigma_{t}$ or $10 \sigma_{t}$, PDTEA-v2 slightly has difficulty in solving dynamic environment. Therefore, when the deviation is large or small, it may negatively affect the exploration.

Table 10: Mean and SD of GD, IGD and HVD indicators of PDTEA-v2 on JY1, JY2 and JY6.

\begin{tabular}{|c|c|c|c|c|c|}
\hline Prob & Indicator & $\sigma=\sigma_{t}$ & $\sigma=0$ & $\sigma=5 \sigma_{t}$ & $\sigma=10 \sigma_{t}$ \\
\hline \multirow{3}{*}{ JY1 } & GD & $1.041 \mathrm{e}-2(1.406 \mathrm{e}-4)$ & $1.185 \mathrm{e}-2(4.381 \mathrm{e}-4) \ddagger$ & $1.492 \mathrm{e}-2(3.337 \mathrm{e}-4) \ddagger$ & $2.409 \mathrm{e}-2(5.928 \mathrm{e}-4) \ddagger$ \\
\hline & IGD & $1.394 \mathrm{e}-2(6.969 \mathrm{e}-5)$ & $1.514 \mathrm{e}-2(3.636 \mathrm{e}-4) \ddagger$ & $1.818 \mathrm{e}-2(2.551 \mathrm{e}-4) \ddagger$ & $2.598 \mathrm{e}-2(4.926 \mathrm{e}-4) \ddagger$ \\
\hline & HVD & $2.123 e-2(2.779 e-4)$ & $2.378 \mathrm{e}-2(8.150 \mathrm{e}-4) \ddagger$ & $2.991 \mathrm{e}-2(7.138 \mathrm{e}-4) \ddagger$ & $4.617 \mathrm{e}-2(1.044 \mathrm{e}-3) \ddagger$ \\
\hline \multirow{3}{*}{ JY2 } & GD & $5.054 e-2(2.522 e-4)$ & $5.150 \mathrm{e}-2(9.933 \mathrm{e}-5) \ddagger$ & $5.169 \mathrm{e}-2(9.116 \mathrm{e}-5) \ddagger$ & $5.523 \mathrm{e}-2(6.955 \mathrm{e}-4) \ddagger$ \\
\hline & IGD & $5.152 \mathrm{e}-2(1.025 \mathrm{e}-4)$ & $5.196 \mathrm{e}-2(1.776 \mathrm{e}-4) \ddagger$ & $5.269 \mathrm{e}-2(7.554 \mathrm{e}-5) \ddagger$ & $5.587 \mathrm{e}-2(2.382 \mathrm{e}-4) \ddagger$ \\
\hline & HVD & $2.716 \mathrm{e}-2(3.823 \mathrm{e}-4)$ & $2.995 \mathrm{e}-2(4.797 \mathrm{e}-4) \ddagger$ & $3.534 \mathrm{e}-2(3.822 \mathrm{e}-4) \ddagger$ & $5.146 \mathrm{e}-2(6.575 \mathrm{e}-4) \ddagger$ \\
\hline \multirow{3}{*}{ JY6 } & GD & $1.726 e+0(1.216 e-1)$ & $1.960 \mathrm{e}+0(8.428 \mathrm{e}-2) \ddagger$ & $2.116 \mathrm{e}+0(9.400 \mathrm{e}-2) \ddagger$ & $2.572 \mathrm{e}+0(4.394 \mathrm{e}-2) \ddagger$ \\
\hline & IGD & $8.617 \mathrm{e}-1(5.390 \mathrm{e}-2)$ & $9.834 \mathrm{e}-1(4.969 \mathrm{e}-2) \ddagger$ & $1.034 \mathrm{e}+0(3.971 \mathrm{e}-2) \ddagger$ & $1.250 \mathrm{e}+0(1.938 \mathrm{e}-2) \ddagger$ \\
\hline & HVD & $4.623 e+0(4.507 \mathrm{e}-1)$ & $5.441 \mathrm{e}+0(4.576 \mathrm{e}-1) \ddagger$ & $5.676 \mathrm{e}+0(3.838 \mathrm{e}-1) \ddagger$ & $7.601 \mathrm{e}+0(2.339 \mathrm{e}-1) \ddagger$ \\
\hline
\end{tabular}

\section{Conclusions and future work}

DMOEAs studies have many real-world applications, like greenhouse control [12] and circular antenna design [62]. In order to effectively deal with DMOPs, we have present a Pareto-based evolutionary algorithm using decomposition and truncation for dynamic multi-objective optimization. The algorithm consists of three parts: a novel mating selection strategy, an efficient environmental selection technique and a dynamic response mechanism. When a change is detected, the loss of population diversity may be of limited coverage. Thus, a dynamic response mechanism including exploration and exploitation strategies is used to adapt to the new environment. Moreover, a mating selection strategy and an environmental selection technique are used to promote the convergence speed of the population. Experimental results demonstrate that, when compared with 
several popular DMOEAs on a number of DMOPs, PDTEA is a very competitive algorithm for dealing with DMOPs, especially for handling three objective problems, a disconnected POF and multimodal problems.

Several extensions are possible for future work:

- Firstly, although PDTEA has great advantages over other algorithms, some new dynamic optimization approaches need to be designed to solve multimodal problems [28]. The reason is that time-changing multimodality is a considerable challenge for an algorithm's performance. Moreover, a large change in severity may require the ability to search the new POS when a change occurs. The proposed dynamic response mechanism can combine with diversity introduction technology to improve the population's diversity.

- Secondly, the DMOEAs have demonstrated the ability to handle constrained DMOPs [63] and solving constrained DMOPs is a prospective research. This paper only considers DMOPs instead of constrained DMOPs. Dealing with constrained DMOPs is our work for the future.

- Last but not least, new dynamic benchmarks [64] and performance metrics 65] are also needed for evaluating the performance of algorithms.

\section{Acknowledgement}

This work was supported by the research projects: the National Natural Science Foundation of China under Grant Nos. 61502408, 61673331, 61772178 and 61403326, the postgraduate research and innovation Project of Hunan Province under Grant No. XDCX2019B057, the MOE (Ministry of Education in China) Liberal Arts and Social Sciences Foundation (Nos. 17YJCZH157) and the Pengcheng Scholar Funded Scheme. 


\section{References}

[1] C. Cruz, J. R. González, D. A. Pelta, Optimization in dynamic environments: a survey on problems, methods and measures, Soft Computing 15 (7) (2011) 1427-1448.

[2] M. Farina, K. Deb, P. Amato, Dynamic multiobjective optimization problems: test cases, approximations, and applications, Evolutionary Computation, IEEE Transactions on 8 (5) (2004) 425-442.

[3] A. Zhou, Y. Jin, Q. Zhang, A population prediction strategy for evolutionary dynamic multiobjective optimization, Cybernetics, IEEE Transactions on 44 (1) (2014) 40-53.

[4] S. Jiang, S. Yang, A steady-state and generational evolutionary algorithm for dynamic multiobjective optimization, IEEE Transactions on Evolutionary Computation PP (99) (2017) 1-1.

[5] S. B. Gee, K. C. Tan, C. Alippi, Solving multiobjective optimization problems in unknown dynamic environments: An inverse modeling approach, IEEE transactions on cybernetics 47 (12) (2017) 4223-4234.

[6] R. Azzouz, S. Bechikh, L. B. Said, A dynamic multi-objective evolutionary algorithm using a change severity-based adaptive population management strategy, Soft Computing 21 (4) (2017) 885-906.

[7] C.-K. Goh, K. C. Tan, A competitive-cooperative coevolutionary paradigm for dynamic multiobjective optimization, Evolutionary Computation, IEEE Transactions on 13 (1) (2009) 103-127.

[8] A. Zhou, Y. Jin, Q. Zhang, B. Sendhoff, E. Tsang, Prediction-based population re-initialization for evolutionary dynamic multi-objective optimization, in: Evolutionary Multi-Criterion Optimization, Springer, 2007, pp. 832-846. 
[9] K. Deb, S. Karthik, et al., Dynamic multi-objective optimization and decision-making using modified nsga-ii: a case study on hydro-thermal power scheduling, in: Evolutionary Multi-Criterion Optimization, Springer, 2007, pp. 803-817.

[10] M. B. Abello, L. T. Bui, Z. Michalewicz, An adaptive approach for solving dynamic scheduling with time-varying number of taskspart ii, in: Evolutionary Computation (CEC), 2011 IEEE Congress on, IEEE, 2011, pp. $1711-1718$.

[11] B. Andres-Toro, J. Giron-Sierra, P. Fernandez-Blanco, J. Lopez-Orozco, E. Besada-Portas, Multiobjective optimization and multivariable control of the beer fermentation process with the use of evolutionary algorithms, Journal of Zhejiang University SCIENCE 5 (4) (2004) 378-389.

[12] Z. Zhang, Multiobjective optimization immune algorithm in dynamic environments and its application to greenhouse control, Applied Soft Computing 8 (2) (2008) 959-971.

[13] E. Tantar, A. Tantar, P. Bouvry, On dynamic multi-objective optimization, classification and performance measures, in: Evolutionary Computation, 2011, pp. 2759-2766.

[14] A. Isaacs, V. Puttige, T. Ray, W. Smith, S. Anavatti, Development of a memetic algorithm for dynamic multi-objective optimization and its applications for online neural network modeling of uavs, in: Neural Networks, 2008. IJCNN 2008.(IEEE World Congress on Computational Intelligence). IEEE International Joint Conference on, IEEE, 2008, pp. 548-554.

[15] L. T. Bui, Z. Michalewicz, An evolutionary multi-objective approach for dynamic mission planning, in: Evolutionary Computation (CEC), 2010 IEEE Congress on, IEEE, 2010, pp. 1-8.

${ }_{940}$ [16] A. R. da Cruz, R. T. Cardoso, R. H. Takahashi, Multiobjective dynamic optimization of vaccination campaigns using convex quadratic approxima- 
tion local search, in: Evolutionary Multi-Criterion Optimization, Springer, 2011, pp. 404-417.

[17] P. P. Wu, D. Campbell, T. Merz, Multi-objective four-dimensional vehicle motion planning in large dynamic environments, Systems, Man, and Cybernetics, Part B: Cybernetics, IEEE Transactions on 41 (3) (2011) 621-634.

[18] K. Deb, Multi-objective optimization using evolutionary algorithms, Vol. 16, John Wiley \& Sons, 2001.

[19] P. Di Barba, Dynamic multiobjective optimization: A way to the shape design with transient magnetic fields, IEEE transactions on magnetics 44 (6) (2008) 962-965.

[20] K. Kim, R. I. McKay, B.-R. Moon, Multiobjective evolutionary algorithms for dynamic social network clustering, in: Proceedings of the 12th annual conference on Genetic and evolutionary computation, ACM, 2010, pp. $1179-1186$.

[21] K. Deb, A. Pratap, S. Agarwal, T. Meyarivan, A fast and elitist multiobjective genetic algorithm: Nsga-ii, Evolutionary Computation, IEEE Transactions on 6 (2) (2002) 182-197.

[22] E. Ziztler, M. Laumanns, L. Thiele, Spea2: Improving the strength pareto evolutionary algorithm for multiobjective optimization, Evolutionary Methods for Design, Optimization, and Control (2002) 95-100.

[23] Q. Zhang, H. Li, Moea/d: A multiobjective evolutionary algorithm based on decomposition, Evolutionary Computation, IEEE Transactions on 11 (6) (2007) 712-731.

965 [24] Y. Wu, Y. Jin, X. Liu, A directed search strategy for evolutionary dynamic multiobjective optimization, Soft Computing (2014) 1-15.

[25] C.-a. Liu, Y. Wang, New evolutionary algorithm for dynamic multiobjective optimization problems, in: Advances in Natural Computation, Springer, 2006, pp. 889-892. 
[26] H. Richter, Detecting change in dynamic fitness landscapes, in: Evolutionary Computation, 2009. CEC'09. IEEE Congress on, IEEE, 2009, pp. 1613-1620.

[27] S. Jiang, M. Kaiser, J. Guo, S. Yang, N. Krasnogor, Less detectable environmental changes in dynamic multiobjective optimisation, in: Proceedings of the Genetic and Evolutionary Computation Conference, ACM, 2018, pp. 673-680.

[28] S. Jiang, S. Yang, Evolutionary dynamic multiobjective optimization: Benchmarks and algorithm comparisons, IEEE transactions on cybernetics 47 (1) (2017) 198-211.

[29] G. Ruan, G. Yu, J. Zheng, J. Zou, S. Yang, The effect of diversity maintenance on prediction in dynamic multi-objective optimization, Applied Soft Computing 58 (2017) 631-647.

[30] R. Chen, K. Li, X. Yao, Dynamic multiobjectives optimization with a changing number of objectives, IEEE Transactions on Evolutionary Computation 22 (1) (2018) 157-171.

[31] M. Helbig, A. P. Engelbrecht, Archive management for dynamic multiobjective optimisation problems using vector evaluated particle swarm optimisation, in: Evolutionary Computation (CEC), 2011 IEEE Congress on, IEEE, 2011, pp. 2047-2054.

[32] I. Hatzakis, D. Wallace, Dynamic multi-objective optimization with evolutionary algorithms: a forward-looking approach, in: Proceedings of the 8th annual conference on Genetic and evolutionary computation, ACM, 2006, pp. 1201-1208.

[33] M. Liu, W. Zeng, Memory enhanced dynamic multi-objective evolutionary algorithm based on decomposition, Ruan Jian Xue Bao/Journal of Software 24 (7) (2013) 1571-1588. 
[34] Z. Peng, J. Zheng, J. Zou, M. Liu, Novel prediction and memory strategies for dynamic multiobjective optimization, Soft Computing 19 (9) (2015) 2633-2653.

[35] A. Muruganantham, K. C. Tan, P. Vadakkepat, Evolutionary dynamic multiobjective optimization via kalman filter prediction, IEEE Trans Cybern 46 (12) (2015) 2862.

[36] Y.-J. Zhang, S.-F. Shao, J. Niyongabo, Cloud hyper mutation particle swarm optimization algorithm based on cloud model, Pattern Recognition and Artificial Intelligence 24 (1) (2011) 90-94.

[37] B. Zheng, A new dynamic multi-objective optimization evolutionary algorithm, in: icnc, IEEE, 2007, pp. 565-570.

[38] R. Liu, W. Zhang, L. Jiao, F. Liu, J. Ma, A sphere-dominance based preference immune-inspired algorithm for dynamic multi-objective optimization, in: Proceedings of the 12th annual conference on Genetic and evolutionary computation, ACM, 2010, pp. 423-430.

[39] A. Díaz-Manríquez, G. T. Pulido, J. G. Ramírez-Torres, Handling dynamic multiobjective problems with particle swarm optimization., in: ICAART (1), 2010, pp. 337-342.

[40] M. Cámara, J. Ortega, F. de Toro, A single front genetic algorithm for parallel multi-objective optimization in dynamic environments, Neurocomputing 72 (16) (2009) 3570-3579.

[41] J. Wei, Y. Wang, Hyper rectangle search based particle swarm algorithm for dynamic constrained multi-objective optimization problems, in: Evolutionary Computation (CEC), 2012 IEEE Congress on, IEEE, 2012, pp. $1-8$.

[42] J. Zheng, G. Yu, Q. Zhu, X. Li, J. Zou, On decomposition methods in interactive user-preference based optimization, Applied Soft Computing 52 (2017) 952-973. 
[50] I. Das, J. E. Dennis, Normal-boundary intersection: A new method for generating the pareto surface in nonlinear multicriteria optimization problems, Siam Journal on Optimization 8 (3) (1998) 631-657.

[51] K. Deb, H. Jain, An evolutionary many-objective optimization algorithm using reference-point-based nondominated sorting approach, part i: Solving problems with box constraints, IEEE Transactions on Evolutionary Computation 18 (4) (2014) 577-601. 
[52] K. Li, Q. Zhang, S. Kwong, M. Li, R. Wang, Stable matching-based selection in evolutionary multiobjective optimization, IEEE Transactions on Evolutionary Computation 18 (6) (2014) 909-923.

[53] A. Zhou, Estimation of distribution algorithms for continuous multiobjective optimization, Ph.D. thesis, University of Essex (2009).

[54] M. Helbig, A. P. Engelbrecht, Benchmarks for dynamic multi-objective optimisation, in: Computational Intelligence in Dynamic and Uncertain Environments (CIDUE), 2013 IEEE Symposium on, IEEE, 2013, pp. 8491.

[55] M. Helbig, A. Engelbrecht, Benchmark functions for cec 2015 special session and competition on dynamic multi-objective optimization, Dept. Comput. Sci., Univ. Pretoria, Pretoria, South Africa, Rep.

[56] J. Zou, Q. Li, S. Yang, H. Bai, J. Zheng, A prediction strategy based on center points and knee points for evolutionary dynamic multi-objective optimization, Applied Soft Computing 61 (2017) 806-818.

[57] K. Li, K. Deb, Q. Zhang, S. Kwong, An evolutionary many-objective optimization algorithm based on dominance and decomposition., IEEE Trans. Evolutionary Computation 19 (5) (2015) 694-716.

[58] Y. Yuan, H. Xu, B. Wang, B. Zhang, X. Yao, Balancing convergence and diversity in decomposition-based many-objective optimizers, IEEE Transactions on Evolutionary Computation 20 (2) (2016) 180-198.

[59] F. Wilcoxon, Individual comparisons by ranking methods, Biometrics bulletin 1 (6) (1945) 80-83.

[60] S. Huband, P. Hingston, L. Barone, L. While, A review of multiobjective test problems and a scalable test problem toolkit, Evolutionary Computation, IEEE Transactions on 10 (5) (2006) 477-506. 
[61] M. Helbig, A. P. Engelbrecht, Analysing the performance of dynamic multiobjective optimisation algorithms, in: Evolutionary Computation (CEC), 2013 IEEE Congress on, IEEE, 2013, pp. 1531-1539.

[62] S. Biswas, D. Bose, S. Das, S. Kundu, Decomposition-based evolutionary multi-objective optimization approach to the design of concentric circular antenna arrays, Progress In Electromagnetics Research 52 (2013) 185-205.

[63] R. Azzouz, S. Bechikh, L. B. Said, W. Trabelsi, Handling time-varying constraints and objectives in dynamic evolutionary multi-objective optimization, Swarm and evolutionary computation 39 (2018) 222-248.

[64] S. B. Gee, K. C. Tan, H. A. Abbass, A benchmark test suite for dynamic evolutionary multiobjective optimization, IEEE transactions on cybernetics 47 (2) (2017) 461-472.

[65] M. Helbig, A. P. Engelbrecht, Performance measures for dynamic multiobjective optimisation algorithms, Information Sciences 250 (2013) 61-81. 